\title{
Mean Square Numerical Methods for Initial Value Random Differential Equations
}

\author{
Magdy A. El-Tawil ${ }^{1^{*}}$, Mohammed A. Sohaly ${ }^{2}$ \\ ${ }^{1}$ Engineering Mathematics Department, Faculty of Engineering, Cairo University, Giza, Egypt \\ ${ }^{2}$ Mathematic Department, Faculty of Science, Mansoura University, Mansoura, Egypt \\ E-mail:magdyeltawil@yahoo.com,m_stat2000@yahoo.com \\ Received April 1, 2011, revised April 30, 2011, accepted May 9, 2011
}

\begin{abstract}
In this paper, the random Euler and random Runge-Kutta of the second order methods are used in solving random differential initial value problems of first order. The conditions of the mean square convergence of the numerical solutions are studied. The statistical properties of the numerical solutions are computed through numerical case studies.
\end{abstract}

Keywords: Random Differential Equations, Mean Square Sense, Second Random Variable, Initial Value Problems, Random Euler Method, Random Runge Kutta-2 Method

\section{Introduction}

Random differential equations (RDE) are defined as differential equations involving random inputs. In recent years, increasing interest in the numerical solution of (RDE) has led to the progressive development of several numerical methods. This paper is interested in studying the following random differential initial value problem (RIVP) of the form:

$$
\frac{\mathrm{d} X}{\mathrm{~d} t}=f(t, X), X\left(t_{0}\right)=X_{0}
$$

Randomness may exist in the initial value or in the differential operator or both. In [1,2], the authors discussed the general order conditions and a global convergence proof is given for stochastic Runge-Kutta methods applied to stochastic ordinary differential equations (SODEs) of Stratonovich type. In $[3,4]$, the authors discussed the random Euler method and the conditions for the mean square convergence of this problem. In [5], the authors considered a very simple adaptive algorithm based on controlling only the drift component of a time step. Platen, E. [6] discussed discrete time strong and weak approximation methods that are suitable for different applications. Other numerical methods are discussed in [7-12].

In this paper the random Euler and random RungeKutta of the second order methods are used to obtain an approximate solution for Equation (1.1). This paper is organized as follows. In Section 2, some important preliminaries are discussed. In Section 3, the existence and uniqueness of the solution of random differential initial value problem is discussed and the convergence of random Euler and random Runge-Kutta of the second order methods is discussed. In Section 4, the statistical properties for the exact and numerical solutions are studied. Section 5 presents the solution of some numerical examples of first order random differential equations using random Euler and random Runge-Kutta of the second order methods showing the convergence of the numerical solutions to the exact ones (if possible). The general conclusions are presented in the end section.

\section{Preliminaries}

\subsection{Mean Square Calculus [13]}

Definition1. Let us consider the properties of a class of real r.v.'s $X_{1}, X_{2}, \cdots, X_{n}$ whose second moments, $E\left\{X_{1}^{2}\right\}, E\left\{X_{2}^{2}\right\}, \cdots$ are finite. In this case, they are called "second order random variables", (2.r.v's).

Definition 2. The linear vector space of second order random variables with inner product, norm and distance, is called an $L_{2}$-space.

A s.p. $\{X(t), t \in T\}$ is called a "second order stochastic process" (2.s.p) if for $t_{1}, t_{2}, \cdots t_{n}$, the r.v's $\left\{X\left(t_{1}\right), X\left(t_{2}\right), \cdots, X\left(t_{n}\right)\right\}$ are elements of $L_{2}$-space. 
A second order s.p. $\{X(t), t \in T\}$ is characterized by $\|X(t)\|^{2}=E\left\{X^{2}(t)\right\}<\infty, t \in T$.

\subsubsection{The Convergence in Mean Square}

A sequence of r.v's $\left\{X_{n}\right\}$ converges in mean square (m.s) to a random variable $X$

$$
\text { if } \lim _{n \rightarrow \infty}\left\|X_{n}-X\right\|=0 \text { i.e. } X_{n} \stackrel{\text { m.s }}{\longrightarrow} X \text { or }
$$

$\lim _{n \rightarrow \infty} X_{n}=X$

where lim is the limit in mean square sense.

\subsubsection{Mean-Square Differentiability}

The random process $\{X(t)\}$ is mean-square differentiable at $\mathrm{t}$ if $\lim _{h \rightarrow 0} \frac{X_{t+h}-X_{t}}{h}$ exists, and is denoted by

$\lim _{h \rightarrow 0} \frac{X_{t+h}-X_{t}}{h}=\dot{X}_{t}$

\section{Random Initial Value Problem (RIVP)}

\subsection{Existence and Uniqueness}

Let us have the random initial value problem

$$
\frac{\mathrm{d} X}{\mathrm{~d} t}=b(t, X), t \in T=\left[t_{0}, t\right], X\left(t_{0}\right)=X_{0}
$$

where $X(t)$ is second order random process. This equation is equivalent to integral equation

$$
X(t)=X_{0}+\int_{t 0}^{t} b(X(s), s) \mathrm{d} s
$$

\section{Theorem (3.1.1)}

If we have the random initial value problem (3.1) and suppose the right-hand side function $b(t, X)$ is continuous and satisfies a mean square (m.s) Lipschitz condition in its second argument:

$$
\|b(t, X)-b(t, Y)\| \leq c\|X-Y\|
$$

where $\mathrm{C}$ is a constant or

$$
\|b(t, X)-b(t, Y)\| \leq c(t)\|X-Y\| \leq c\|X-Y\|
$$

where $c(t)$ is a continuous function \{because in every finite interval $c(t) \leq$ constant $\}$.

then the solution of Equation (3.1) exists and is unique.

The proof

The existence can be proved by using successive approximations. Let

$$
X_{t}^{0}=X_{0}
$$

and for $n \geq 1$

$$
X_{t}^{n}=X_{0}+\int_{t 0}^{t} b\left(X_{s}^{n-1}, s\right) \mathrm{d} s .
$$

For $n=1$ we obtain:

$$
\left\|X_{t}^{(1)}-X_{t}^{(0)}\right\|=\left\|\int_{t 0}^{t} b\left(s, X_{0}\right) \mathrm{d} s\right\| \leq k \cdot\left|t-t_{0}\right|
$$

where

$$
\|b(t, X)\| \leq k
$$

For $n>1$ we obtain:

$$
\begin{aligned}
\left\|X_{t}^{(n)}-X_{t}^{(n-1)}\right\| & =\left\|\int_{t 0}^{t} b\left(s, X_{s}^{n-1}\right)-b\left(s, X_{s}^{n-2}\right) \mathrm{d} s\right\| \\
& \leq \int_{t 0}^{t} c \cdot\left\|X_{s}^{(n-1)}-X_{s}^{(n-2)}\right\| \mathrm{d} s
\end{aligned}
$$

Successively, we can obtain the following:

$$
\begin{aligned}
\left\|X_{t}^{(n)}-X_{t}^{(n-1)}\right\| & \leq \int_{t_{0}}^{t} c\left\|X_{s}^{n-1}-X_{s}^{n-2}\right\| \mathrm{d} s \\
& \leq \int_{t_{0}}^{t} c \int_{t_{0}}^{t} c\left\|X_{s}^{n-2}-X_{s}^{n-3}\right\| \mathrm{d} s \mathrm{~d} s \leq c^{3} \int_{t_{0}}^{t} \int_{t_{0}}^{t} \int_{0}^{t}\left\|X_{s}^{n-3}-X_{s}^{n-4}\right\| \mathrm{d} s \mathrm{~d} s \mathrm{~d} s \\
& \leq c^{n-1} \int_{t_{0}}^{t} \int_{t_{0}}^{t} \cdots \int_{t_{0}}^{t}\left\|X_{s}^{1}-X_{s}^{0}\right\| \mathrm{d} s \cdots \mathrm{d} s \leq c^{n-1} \int_{t_{0}}^{t} \int_{t_{0}}^{t} \cdots \int_{t_{0}}^{t} k\left|s-t_{0}\right| \mathrm{d} \cdots \mathrm{d} s .
\end{aligned}
$$

Hence

$$
\left.\left\|X^{n}-X^{n-1}\right\| \leq k c^{n-1} \int_{t_{0}}^{t}\left[\int_{t_{0}}^{t} \cdots \int_{t_{0}}^{t}\left[\int_{t_{0}}^{t}\left|s-t_{0}\right| \mathrm{d} s\right] \mathrm{d} s\right] \cdots \mathrm{d} s\right] \mathrm{d} s=k c^{n-1} \frac{\left|t-t_{0}\right|^{n}}{n !}
$$

Since:

$$
\sum_{n=1}^{\infty} k \cdot c^{(n-1)} \frac{\left|t-t_{0}\right|}{n !}=\frac{k}{c} \mathrm{e}^{c \mid t-t_{0} \|} \text { is convergent for finite } \mathrm{t},
$$

hence we can have the following 
$\left\|X_{t}^{1}-X_{t}^{0}\right\|+\left\|X_{t}^{2}-X_{t}^{1}\right\|+\cdots+\left\|X_{t}^{n-1}-X_{t}^{n-2}\right\|+\cdots \leq\left(\frac{k\left|t-t_{0}\right|}{1 !}\right)+\left(\frac{k c\left|t-t_{0}\right|^{2}}{2 !}\right)+\cdots+\left(\frac{k c^{n-2}\left|t-t_{0}\right|^{n-1}}{(n-1) !}\right)+\cdots=\sum_{n=1}^{\infty} k \cdot c^{(n-1)} \frac{\left|t-t_{0}\right|^{n}}{n !}$

Accordingly,

$$
\left\|X_{t}^{1}-X_{t}^{0}+X_{t}^{2}-X_{t}^{1}+\cdots+X_{t}^{n-1}-X_{t}^{n-2}+X_{t}^{n}-X_{t}^{n-1}\right\|=\lim _{n \rightarrow \infty}\left\|X_{t}^{n}-X_{t}^{0}\right\|=\left\|X_{t}\right\|
$$

Hence:

$\left\|X_{t}^{1}-X_{t}^{0}+X_{t}^{2}-X_{t}^{1}+\cdots X_{t}^{n-1}-X_{t}^{n-2}+X_{t}^{n}-X_{t}^{n-1}\right\| \leq\left\|X_{t}^{1}-X_{t}^{0}\right\|+\left\|X_{t}^{2}-X_{t}^{1}\right\|+\cdots+\left\|X_{t}^{n-1}-X_{t}^{n-2}\right\|+\left\|X^{n}-X^{n-1}\right\|=\frac{k}{c} \mathrm{e}^{c \mid\left\|t t_{0}\right\|}$

This yield $\lim _{n \rightarrow \infty}\left\|X_{t}^{n}-X_{t}^{0}\right\|=\left\|X_{t}\right\| \leq \frac{k}{c} \mathrm{e}^{c \mid t-t_{0} \|}$

Then $\lim _{n \rightarrow \infty} X_{n}$ exists. i.e.

$$
X_{t}=\lim _{n \rightarrow \infty} X_{t}^{n}
$$

Since $X_{t}^{n}$ is the general solution of Equation (3.6) and $X_{t}$ is the general solution of Equation (3.2).

To prove the uniqueness of the solution, let $X_{t}$ is a solution of the initial-value problem (3.1), or, which is the same, of the integral Equation (3.2), and $Y_{t}$ is the solution of

$$
\frac{\mathrm{d} y}{\mathrm{~d} t}=b(Y(t), t), t \in T=\left[t_{0}, t\right], Y\left(t_{0}\right)=Y_{0}
$$

to prove the uniqueness of the solution we want to prove that

$$
X_{t}=Y_{t} .
$$

By subtraction (3.2) and the corresponding integral equation for $Y_{t}$

$$
X_{t}-Y_{t}=X_{0}-Y_{0}+\int_{t_{0}}^{t}\left(b\left(X_{s}, s\right)-b\left(Y_{s}, s\right)\right) \mathrm{d} s
$$

Since $X_{0}=Y_{0}$ then:

$$
\left\|X_{t}-Y_{t}\right\| \leq \int_{t_{0}}^{t} c \cdot\left\|X_{s}-Y_{s}\right\| \mathrm{d} s
$$

i.e; $U^{t} \leq \int_{t 0}^{t} c \cdot U_{s} \mathrm{~d} s$

where $U_{t}=\left\|X_{t}-Y_{t}\right\|$.

From Equation (3.17) we have:

$$
\left\|U_{t}\right\| \leq c\left|t-t_{0}\right|\left\|U_{t}\right\|
$$

Note that: at $t=t_{0}$ we obtain $\left\|U_{0}\right\| \leq 0$ then: $\left\|U_{0}\right\|=0$

From (3.19) $c$ must satisfy the following condition:

$$
c \geq \frac{1}{\left|t-t_{0}\right|}
$$

which is in contradiction with being an independent free constant, hence the only solution of the integral Equation (3.17) is

$$
U^{t}=0
$$

Hence $X_{t}=Y_{t}$ i.e., the solution of Equation (3.1) exists and is unique.

\subsection{The Convergence of Euler Scheme for Random Differential Equations in (m.s.) Sense}

Let us have the random differential equation

$$
\dot{X}(t)=f(X(t), t), t \in T=\left[t_{0}, t_{1}\right], X\left(t_{0}\right)=X_{0}
$$

where $X_{0}$ is a random variable and the unknown $X(t)$ as well as the right-hand side $f(X, t)$ are stochastic processes defined on the same probability space.

Definitions [6,7]

- Let g: $T \rightarrow L_{2}$ is an m.s. bounded function and let $h$ $>0$ then

The "m.s. modulus of continuity of $\mathrm{g}$ " is the function

$$
W(g, h)=\sup _{\left|t-t^{*}\right| \leq h}\left\|g(t)-g\left(t^{*}\right)\right\|, t, t^{*} \in T
$$

- The function $g$ is said to be m.s uniformly continuous in $T$ if:

$$
\lim _{h \rightarrow 0} W(g, h)=0
$$

Note that:

(The limit depends on $\mathrm{h}$ because $\mathrm{g}$ is defined at every $\mathrm{t}$ so we can write $W(g, h)=W(h))$

In the problem (3.22), we find that the convergence of this problem depends on the right hand side (i.e. $f(X(t), t)$ then we want to apply the previous definition on $f(X(t), t)$ hence:

Let $f(X(t), t)$ be defined on $S \times T$ where $S$ is bounded set in $L_{2}$

Then we say that $f$ is "randomly bounded uniformly continuous" in $\mathrm{S}$, if

$$
\lim _{h \rightarrow 0} W(f(x, .), h)=0
$$


(note that $W(f(X(), h))=.W(h))$

\subsubsection{Random Mean Value Theorem for Stochastic Processes}

The aim of this section is to establish a relationship between the increment $X(t)-X\left(t_{0}\right)$ of a 2-s.p. and its m.s. derivative $\dot{X}(\xi)$ for some $\xi$ lying in the interval $\left[t_{0}, t\right]$ for $t>t_{0}$. This result will be used in the next section to prove the convergence of the random Euler method.

\section{Lemma (3.3.2) [6,7]}

Let $Y(t)$ is a 2-s.p., m.s. continuous on interval $T=\left[t_{0}, t\right]$. Then, there exists $\xi \in\left[t_{0}, t\right]$ such that

$$
\int_{t_{0}}^{t} Y(s) \mathrm{d} s=Y(\xi)\left(t-t_{0}\right), t_{0}<t_{1}<t
$$

\section{The proof}

Since $Y(t)$ is m.s. continuous, the integral process $\int_{t_{0}}^{t} Y(s) \mathrm{d} s$ is well defined and the correlation function $\Gamma_{y}(r, s)$ is well defined, is a deterministic continuous function on $T \times T$.

For each fixed $\mathrm{r}$, the function $\Gamma_{y}(r, s)$ is continuous and by the classic mean value theorem for integrals, it follows that:

$$
\begin{aligned}
& \int_{t_{0}}^{t} \Gamma_{y}(r, s) \mathrm{d} s=\Gamma_{y}(r, \xi)\left(t-t_{0}\right) \\
& \forall \xi \in\left[t_{0}, t\right]
\end{aligned}
$$

Note that by definition of $\Gamma_{y}(r, s)$ expression (3.26) can be written in the form

$$
\int_{t_{0}}^{t} E[y(r) y(s)] \mathrm{d} s=E[y(r) y(\xi)]\left(t-t_{0}\right)
$$

Since $\Gamma_{y}(r, s)=E[y(r) y(s)]$

We must prove that for the value $\xi$ satisfying (3.26) one get:

$$
\begin{aligned}
& \left\|\int_{t_{0}}^{t} y(s) \mathrm{d} s-y(\xi)\left(t-t_{0}\right)\right\|^{2} \\
& =E\left[\left(\int_{t_{0}}^{t} y(s) \mathrm{d} s-y(\xi)\left(t-t_{0}\right)\right)^{2}\right]=0
\end{aligned}
$$

\section{The proof of (3.27)}

As

$$
\begin{aligned}
& E\left[\left(\int_{t_{0}}^{t} y(s) \mathrm{d} s-y(\xi)\left(t-t_{0}\right)\right)^{2}\right] \\
& =E\left[\left(\int_{t_{0}}^{t} y(s) \mathrm{d} s\right)^{2}\right]-2 E\left[\left(\int_{t_{0}}^{t} y(s) \mathrm{d} s\right) y(\xi)\right]\left(t-t_{0}\right) \\
& \quad+E\left[y(\xi)^{2}\left(t-t_{0}\right)^{2}\right]
\end{aligned}
$$

and since:

$$
E\left[\left(\int_{t_{0}}^{t} y(s) \mathrm{d} s\right)^{2}\right]=\int_{t_{0}}^{t} \int_{t_{0}}^{t} E[y(s) y(r)] \mathrm{d} r \mathrm{~d} s
$$

then by substituting in (3.28)

$$
\begin{aligned}
E & {\left[\left(\int_{t_{0}}^{t} y(s) \mathrm{d} s-y(\xi)\left(t-t_{0}\right)\right)^{2}\right] } \\
= & \int_{t_{0}}^{t} \int_{t_{0}}^{t} E[y(s) y(r)] \mathrm{d} r \mathrm{~d} s-\int_{t_{0}}^{t} E[y(s) y(\xi) \mathrm{d} s)\left(t-t_{0}\right) \\
& -\int_{t_{0}}^{t} E[y(s) y(\xi) \mathrm{d} s)\left(t-t_{0}\right)+E\left[y(\xi) y(\xi)\left(t-t_{0}\right)^{2}\right.
\end{aligned}
$$

And since:

$$
\int_{t_{0}}^{t} E[y(r) y(s)] \mathrm{d} s=E[y(r) y(\xi)]\left(t-t_{0}\right)
$$

then by substituting in (3.28) we have:

$$
\begin{array}{rl}
E & E\left[\left(\int_{t_{0}}^{t} y(s) \mathrm{d} s-y(\xi)\left(t-t_{0}\right)\right)^{2}\right] \\
= & \int_{t_{0}}^{t} E[y(s) y(\xi) \mathrm{d} s)\left(t-t_{0}\right)-\int_{t_{0}}^{t} E[y(s) y(\xi) \mathrm{d} s)\left(t-t_{0}\right) \\
& -E\left[y(\xi) y(\xi)\left(t-t_{0}\right)^{2}+E\left[y(\xi) y(\xi)\left(t-t_{0}\right)^{2}=0\right.\right.
\end{array}
$$

i.e. $\left\|\int_{t_{0}}^{t} y(s) \mathrm{d} s-y(\xi)\left(t-t_{0}\right)\right\|^{2}=0 \quad$ we obtain

$\int_{t_{0}}^{t} y(s) \mathrm{d} s=y(\xi)\left(t-t_{0}\right)$

Theorem (3.3.1) $[6,7]$

Let $X(s)$ be a m.s. differentiable 2-s.p. in $\left[t_{0}, t_{1}\right]$ and m.s. continuous in $T=\left[t_{0}, t\right]$. Then, there exists $\xi \in\left[t_{0}, t_{1}\right]$ such that $X(t)-X\left(t_{0}\right)=\dot{X}(\xi)\left(t-t_{0}\right)$, $t_{0}<t_{1}<t$

\section{The proof}

The result is a direct consequence of Lemma (3.3.2) applied to the 2-s.p. $Y(t)=\dot{X}(t)$

$$
\int_{t_{0}}^{t} \dot{X}(s) \mathrm{d} s=\dot{X}(\xi)\left(t-t_{0}\right)
$$

And the integral formula

$$
\int_{t_{0}}^{t} \dot{X}(s) \mathrm{d} s=X(t)-X\left(t_{0}\right)
$$

The proof of (3.30)

Let $X(t)$ be a m.s. differentiable on $\mathrm{T}$ and let the ordinary function $f(t, s)$ be continuous on $T \times T$ whose partial derivative $\frac{\partial f(t, s)}{\partial s}$ exist

If $Y(t)=\int_{a}^{t} f(t, s) \dot{X}(s) \mathrm{d} s$

Then

$$
Y(t)=\left.f(t, s) X(s)\right|_{a} ^{t}-\int_{a}^{t} \frac{\partial f(t, s)}{\partial s} X(s) \mathrm{d} s
$$

Let $f(t, s)=1$ in Equations (3.31) and (3.32) we 
have the useful result that:

If $\dot{X}(t)$ is m.s. Riemann integrable on T then:

$$
\int_{a}^{t} \dot{X}(s) d s=X(t)-X(a),[a, t] \subset T
$$

Then we have:

$$
X(t)-X\left(t_{0}\right)=\dot{X}(\xi)\left(t-t_{0}\right)
$$

\subsubsection{The Convergence of Random Euler Scheme}

In this section we are interested in the mean square convergence, in the fixed station sense, of the random Euler method defined by

$$
X_{n+1}=X_{n}+h f\left(X_{n}, t_{n}\right), X\left(t_{0}\right)=X_{0}, n \geq 0
$$

where $X_{n}$ and $f\left(X_{n}, t_{n}\right)$ are 2-r.v.'s,$h=t_{n}-t_{n-1}$, $t_{n}=t_{0}+n h$ and $f: S \times T \rightarrow L_{2}, \quad S \subset L_{2}$ satisfies the following conditions:

$\mathrm{C} 1$ : $f(X, t)$ is randomly bounded uniformly continuous,

C2: $f(X, t)$ satisfies the m.s. Lipschitz condition

$$
\|f(x, t)-f(y, t)\| \leq k(t)\|x-y\|
$$

where

$$
\int_{t_{0}}^{t_{1}} k(t) \leq \infty
$$

Note that under hypothesis $\mathrm{C} 1$ and $\mathrm{C} 2$, we are interested in the m.s. convergence to zero of the error

$$
e_{n}=X_{n}-X(t)
$$

where $X(t)$ is the theoretical solution 2-s.p. of the problem (3.22), $t=t_{n}=t_{0}+n h$.

Taking into account (3.22), and Theorem (3.3.1), one gets,

Since from (3.22) we have at $t=t_{\xi}$ then

$$
\dot{X}\left(t_{\xi}\right)=f\left(x\left(t_{\xi}\right), t_{\xi}\right)
$$

Note $\xi \in\left[t_{0}, t_{1}\right]$ and we can use $\xi$ instead of $t_{\xi}$ and from Theorem (3.3.1) at $t=t_{\xi}$ then we have:

$$
\begin{aligned}
X\left(t_{\xi}\right)-X\left(t_{0}\right)=\dot{X}(t)\left(t_{\xi}-t_{0}\right) \text { then } \\
\quad X\left(t_{\xi}\right)-X\left(t_{0}\right)=f\left(X\left(t_{\xi}, t_{\xi}\right)\right)\left(t_{\xi}-t_{0}\right)
\end{aligned}
$$

Note that we deal with the interval $\left(t_{n}, t_{n+1}\right) \ni t_{\xi}$ $\in\left(t_{n}, t_{n+1}\right)$ and hence $t_{0}$ was the starting in the problem (3.22) and here $t_{n}$ is the starting and since Euler method deal with solution depend on previous solution and if we have $X\left(t_{n}\right)$ instead of $X\left(t_{0}\right)$ then we can use $X\left(t_{n+1}\right)$ instead of $X\left(t_{\xi}\right)$.

Then the final form of the problem (3.22) is

$$
X\left(t_{n+1}\right)=X\left(t_{n}\right)+h f\left(X\left(t_{\xi}, t_{\xi}\right)\right) \text {, for some }
$$

$$
t_{\xi} \in\left(t_{n}, t_{n+1}\right)
$$

Now we have the solution of problem (3.22) is $X\left(t_{n}\right)$ At $t=t_{n}$ then $X\left(t_{n}\right)=X(t)$ and the solution of Euler method (3.33) is $X_{n}$

Then we can define the error

$$
\begin{gathered}
e_{n}=X_{n}-X\left(t_{n}\right) \\
e_{n}=X_{n}-X(t)
\end{gathered}
$$

By (3.33) and (3.36) it follows that

$$
\begin{aligned}
& X_{n+1}-X\left(t_{n+1}\right) \\
& =X_{n}+h f\left(X_{n}, t_{n}\right)-X\left(t_{n}\right)-h f\left(X\left(t_{\xi}, t_{\xi}\right)\right)
\end{aligned}
$$

This implies

$$
e_{n+1}=X_{n}-X\left(t_{n}\right)+h\left\{f\left(X_{n}, t_{n}\right)-f\left(X\left(t_{\xi}\right), t_{\xi}\right)\right\}
$$

Hence

$$
\begin{aligned}
\left\|e_{n+1}\right\| & =\left\|X_{n}-X\left(t_{n}\right)+h\left\{f\left(X_{n}, t_{n}\right)-f\left(X\left(t_{\xi}\right), t_{\xi}\right)\right\}\right\| \\
& \leq\left\|X_{n}-X\left(t_{n}\right)\right\|+h\left\|f\left(X\left(t_{\xi}\right), t_{\xi}\right)-f\left(X_{n}, t_{n}\right)\right\|
\end{aligned}
$$

Since:

$$
\begin{aligned}
\| & f\left(X\left(t_{\xi}\right), t_{\xi}\right)-f\left(X_{n}, t_{n}\right) \| \\
= & \| f\left(X\left(t_{\xi}\right), t_{\xi}\right)-f\left(X\left(t_{\xi}\right), t_{n}\right)+f\left(X\left(t_{\xi}\right), t_{n}\right) \\
& +f\left(X\left(t_{n}\right), t_{n}\right)-f\left(X\left(t_{n}\right), t_{n}\right)-f\left(X_{n}, t_{n}\right) \| \\
\leq & \left\|f\left(X\left(t_{\xi}\right), t_{\xi}\right)-f\left(X\left(t_{\xi}\right), t_{n}\right)\right\| \\
& +\left\|f\left(X\left(t_{\xi}\right), t_{n}\right)-f\left(X\left(t_{n}\right), t_{n}\right)\right\| \\
& +\left\|f\left(X\left(t_{n}\right), t_{n}\right)-f\left(X_{n}, t_{n}\right)\right\|
\end{aligned}
$$

Since the theoretical solution $X$ is m.s. bounded in $\left[t_{0}, t_{1}\right], \quad \sup \|X(t)\| \leq M<\infty$ and

Under hypothesis $\mathrm{C} 1, \mathrm{C} 2 \mathrm{We}$ obtain

- $\left\|f\left(X\left(t_{\xi}\right), t_{\xi}\right)-f\left(X\left(t_{\xi}\right), t_{n}\right)\right\|=w(h)$

- $\left\|f\left(X\left(t_{\xi}\right), t_{n}\right)-f\left(X\left(t_{n}\right), t_{n}\right)\right\| \leq k\left(t_{n}\right) M h$

Since $k\left(t_{n}\right)$ is Lipschitz constant (from C2) and from Theorem (3.3.1) we have $X(t)-X\left(t_{0}\right)=\dot{X}(\xi)\left(t-t_{0}\right)$ and note that the two points are $X\left(t_{\xi}\right)$ and $X\left(t_{n}\right)$ in $\left.{ }^{*}\right)$ then we have:

$$
\left\|X\left(t_{\xi}\right)-X\left(t_{n}\right)\right\|=\|\dot{X}(\xi)\|\left|t_{\xi}-t_{n}\right| \leq M h
$$

Since $\left|t_{\xi}-t_{n}\right|=h$ and $M=\sup _{t_{0} \leq t \leq t_{1}}\|\dot{X}(t)\|$

- $\left\|f\left(X\left(t_{n}\right), t_{n}\right)-f\left(X_{n}, t_{n}\right)\right\|$ $\leq k\left(t_{n}\right)\left\|X\left(t_{n}\right)-X_{n}\right\|=k\left(t_{n}\right)\left\|e_{n}\right\|$ 
Then by substituting in (3.38) we have

$$
\left\|f\left(X\left(t_{\xi}\right), t_{\xi}\right)-f\left(X_{n}, t_{n}\right)\right\| \leq w(h)+k\left(t_{n}\right) M h+k\left(t_{n}\right)\left\|e_{n}\right\|
$$

Then by substituting in (3.37) we have

$$
\begin{aligned}
& \left\|e_{n+1}\right\| \leq\left\|e_{n}\right\|+h\left[w(h)+k\left(t_{n}\right) M h+k\left(t_{n}\right)\left\|e_{n}\right\|\right]=\left(1+k\left(t_{n}\right) h\right)\left\|e_{n}\right\|+h\left[w(h)+k\left(t_{n}\right) M h\right] \\
& \leq\left(1+K\left(t_{n}\right) h\right)\left[\left(1+k\left(t_{n}\right) h\right)\left\|e_{n-1}\right\|+h\left[w(h)+k\left(t_{n}\right) M h\right]+h\left[w(h)+k\left(t_{n}\right) M h\right]\right. \\
& =\left(1+K\left(t_{n}\right) h\right)^{2}\left\|e_{n-1}\right\|+h\left[w(h)+k\left(t_{n}\right) M h\right]\left[1+\left(1+k\left(t_{n}\right) h\right)\right] \\
& \leq\left(1+K\left(t_{n}\right) h\right)^{3}\left\|e_{n-2}\right\|+h\left[w(h)+k\left(t_{n}\right) M h\right]\left[1+\left(1+k\left(t_{n}\right) h\right)+\left(1+k\left(t_{n}\right) h\right)^{2}\right] \\
& \leq\left(1+K\left(t_{n}\right) h\right)^{n+1}\left\|e_{0}\right\|+h\left[w(h)+k\left(t_{n}\right) M h\right]\left[1+\left(1+k\left(t_{n}\right) h\right)+\left(1+k\left(t_{n}\right) h\right)^{2}+\cdots+\left(1+k\left(t_{n}\right) h\right)^{n}\right]
\end{aligned}
$$

Since:

$\left[1+\left(1+k\left(t_{n}\right) h\right)+\left(1+k\left(t_{n}\right) h\right)^{2}+\cdots+\left(1+k\left(t_{n}\right) h\right)^{n}\right]$

is geometrical sequence.

Then:

$$
\begin{aligned}
& {\left[1+\left(1+k\left(t_{n}\right) h\right)+\left(1+k\left(t_{n}\right) h\right)^{2}+\cdots+\left(1+k\left(t_{n}\right) h\right)^{n}\right]} \\
& =\frac{\left(1+k\left(t_{n}\right) h\right)^{n}-1}{k\left(t_{n}\right) h}
\end{aligned}
$$

Then we get

$$
\begin{aligned}
\left\|e_{n+1}\right\| \leq & \left(1+K\left(t_{n}\right) h\right)^{n+1}\left\|e_{0}\right\| \\
& +\left[w(h)+k\left(t_{n}\right) M h\right] \frac{\left[\left(1+k\left(t_{n}\right) h\right)^{n}-1\right]}{k\left(t_{n}\right)}
\end{aligned}
$$

Taking into account that $e_{0}=0$ where $e_{0}=X_{0}-X\left(t_{0}\right)=0$.

$$
\begin{aligned}
\left\|e_{n+1}\right\| \leq & {\left[w(h)+k\left(t_{n}\right) M h\right] \frac{\left[\left(1+k\left(t_{n}\right) h\right)^{n}-1\right]}{k\left(t_{n}\right)} } \\
\lim _{h \rightarrow 0}\left\|e_{n+1}\right\| \leq & \lim _{h \rightarrow 0}\left[w(h)+k\left(t_{n}\right) M h\right] \frac{\left[\left(1+k\left(t_{n}\right) h\right)^{n}-1\right]}{k\left(t_{n}\right)} \\
= & \lim _{h \rightarrow 0} \frac{\left[w(h)+k\left(t_{n}\right) M h\right]}{k\left(t_{n}\right)}\left[\left(1+K\left(t_{n}\right) h\right)^{n}\right] \\
& -\lim _{h \rightarrow 0} \frac{\left[w(h)+k\left(t_{n}\right) M h\right]}{k\left(t_{n}\right)}
\end{aligned}
$$

Note that:
The term: $\lim _{h \rightarrow 0} \frac{\left[w(h)+k\left(t_{n}\right) M h\right]}{k\left(t_{n}\right)}=0$ as

$$
h \rightarrow 0 \quad(\ni \quad w(h) \rightarrow 0 \text { as } h \rightarrow 0)
$$

And the second term:

$$
\lim _{h \rightarrow 0} \frac{\left[w(h)+k\left(t_{n}\right) M h\right]}{k\left(t_{n}\right)}\left[\left(1+K\left(t_{n}\right) h\right)^{n}\right]
$$

we have:

$$
\begin{aligned}
& \lim _{h \rightarrow 0} \frac{\left[w(h)+k\left(t_{n}\right) M h\right]}{k\left(t_{n}\right)}\left[\left(1+K\left(t_{n}\right) h\right)^{n}\right] \\
& =\lim _{h \rightarrow 0} \frac{\left[w(h)+k\left(t_{n}\right) M h\right]}{k\left(t_{n}\right)} \lim _{h \rightarrow 0}\left[\left(1+K\left(t_{n}\right) h\right)^{n}\right]
\end{aligned}
$$

The first limit in (3.42) equal zero and:

The computation of $\lim _{h \rightarrow 0}\left[\left(1+K\left(t_{n}\right) h\right)^{n}\right]$ as follows:

Let $y=\lim _{h \rightarrow 0}\left[\left(1+K\left(t_{n}\right) h\right)^{n}\right]$ then by tacking the logarithm of the two sides we have:

$$
\begin{aligned}
\ln y & =\ln _{h \rightarrow 0}\left[\left(1+K\left(t_{n}\right) h\right)^{n}\right] \\
& =\lim _{h \rightarrow 0} \ln \left[\left(1+K\left(t_{n}\right) h\right)^{n}\right] \\
& =\lim _{h \rightarrow 0} n \ln \left[\left(1+K\left(t_{n}\right) h\right)\right] \\
& =\lim _{h \rightarrow 0} \frac{t_{n}-t_{0}}{h} \ln \left[\left(1+K\left(t_{n}\right) h\right)\right] \\
& =\lim _{h \rightarrow 0} \frac{t_{n}-t_{0}}{h} \ln \left[\left(1+K\left(t_{n}\right) h\right)\right] \\
& =\lim _{h \rightarrow 0} \frac{\left(t_{n}-t_{0}\right)\left[\ln \left(1+k\left(t_{n}\right) h\right)\right]}{h}
\end{aligned}
$$

By using the (L'Hospital's Rule): 


$$
\begin{aligned}
& \lim _{h \rightarrow 0} \frac{\left(t_{n}-t_{0}\right)\left[\ln \left(1+k\left(t_{n}\right) h\right)\right]}{h} \\
& =\lim _{h \rightarrow 0} \frac{\left(t_{n}-t_{0}\right) \frac{1}{\left(1+k\left(t_{n}\right) h\right)} k\left(t_{n}\right)}{1} \\
& =\lim _{h \rightarrow 0} \frac{\left(t_{n}-t_{0}\right) k\left(t_{n}\right)}{1+k\left(t_{n}\right) h}=\left(t-t_{0}\right) k(t)
\end{aligned}
$$

Then $\ln y=\left(t-t_{0}\right) k\left(t_{n}\right)$ which implies that $y=\mathrm{e}^{\left(t-t_{0}\right) k\left(t_{n}\right)}$ hence

$$
\lim _{h \rightarrow 0}\left[\left(1+K\left(t_{n}\right) h\right)^{n}\right]=\mathrm{e}^{\left(t-t_{0}\right) k\left(t_{n}\right)}
$$

By substituting in (3.42):

$$
\begin{aligned}
& \lim _{h \rightarrow 0} \frac{\left[w(h)+k\left(t_{n}\right) M h\right]}{k\left(t_{n}\right)}\left[\left(1+K\left(t_{n}\right) h\right)^{n}\right] \\
& =0 \times \mathrm{e}^{\left(t-t_{0}\right) k\left(t_{n}\right)}=0
\end{aligned}
$$

By substituting from (3.44) and (3.42) in (3.40) hence $\lim _{h \rightarrow 0}\left\|e_{n+1}\right\| \rightarrow 0$ i.e., $\left\{e_{n}\right\}$ converge in m.s to zero as $h \rightarrow 0$ hence $X_{n} \stackrel{m \cdot s}{\longrightarrow} X\left(t_{n}\right)=X(t)$.

\subsection{The Convergence of Runge-Kutta of Second Order Scheme for Random Differential Equations in Mean Square Sense}

In this section we are interested in the mean square convergence, in the fixed station sense, of the random Runge-Kutta of second order method defined by

$$
\begin{aligned}
& X_{n+1}=X_{n}+\frac{h}{2}\left[f\left(X_{n}, t_{n}\right)+f\left(X_{n}+f\left(X_{n}, t_{n}\right), t_{n+1}\right),\right. \\
& X\left(t_{0}\right)=X_{0}, n \geq 0
\end{aligned}
$$

where $X_{n}$ and $f\left(X_{n}, t_{n}\right)$ are 2-r.v.'s, $h=t_{n}-t_{n-1}$, $t_{n}=t_{0}+n h$ and $f: S \times T \rightarrow L_{2}, \quad S \subset L_{2}$ satisfies the following conditions:

C1: $f(X, t)$ is randomly bounded uniformly continuous,

C2: $f(X, t)$ satisfies the m.s. Lipschitz condition

$$
\|f(x, t)-f(y, t)\| \leq k(t)\|x-y\|
$$

where

$$
\int_{t_{0}}^{t_{1}} k(t) \leq \infty
$$

Note that under hypothesis C1 and C2, we are interested in the m.s. convergence to zero of the error

$$
e_{n}=X_{n}-X(t)
$$

where $X(t)$ is the theoretical solution 2-s.p. of the problem (3.22), $t=t_{n}=t_{0}+n h$.

Taking into account (3.22), and Theorem (3.3.1), one gets,

Since from (3.22) we have at $t=t_{\xi}$ then

$$
\dot{X}\left(t_{\xi}\right)=f\left(x\left(t_{\xi}\right), t_{\xi}\right)
$$

Note $\xi \in\left[t_{0}, t_{1}\right]$ and we can use $\xi$ instead of $t_{\xi}$ And from Theorem (3.3.1) at $t=t_{\xi}$ then we obtain

$$
\begin{aligned}
& X\left(t_{\xi}\right)-X\left(t_{0}\right)=\dot{X}(t)\left(t_{\xi}-t_{0}\right) \Rightarrow \\
& X\left(t_{\xi}\right)-X\left(t_{0}\right)=f\left(X\left(t_{\xi}, t_{\xi}\right)\left(t_{\xi}-t_{0}\right)\right)
\end{aligned}
$$

Note that we deal with the interval $\left(t_{n}, t_{n+1}\right) \ni t_{\xi}$ $\in\left(t_{n}, t_{n+1}\right)$ and hence $t_{0}$ was the starting in the problem (3.22) and here $t_{n}$ is the starting and since Euler method deal with solution depend on previous solution and if we have $X\left(t_{n}\right)$ instead of $X\left(t_{0}\right) \Rightarrow$ we can use $X\left(t_{n+1}\right)$ instead of $X\left(t_{\xi}\right)$ then the final form of the problem (3.22) is

$X\left(t_{n+1}\right)=X\left(t_{n}\right)+h f\left(X\left(t_{\xi}, t_{\xi}\right)\right)$, for some $t_{\xi} \in\left(t_{n}, t_{n+1}\right)$

Now we have the solution of problem (3.22) is $X\left(t_{n}\right)$

At $t=t_{n}$ then $X\left(t_{n}\right)=X(t)$ and the solution of Runge-Kutta of 2 order method (3.45) is $X_{n}$

Then we can define the error

$$
e_{n}=X_{n}-X(t)
$$

By (3.45) and (3.48) it follows that

$$
\begin{aligned}
& X_{n+1}-X\left(t_{n+1}\right) \\
& =X_{n}+\frac{h}{2}\left[f\left(X_{n}, t_{n}\right)+f\left(X_{n}+f\left(X_{n}, t_{n}\right), t_{n+1}\right)\right]-X\left(t_{n}\right) \\
& \quad-\frac{h}{2} f\left(X\left(t_{\xi}\right), t_{\xi}\right)-\frac{h}{2} f\left(X\left(t_{\xi}\right), t_{\xi}\right)
\end{aligned}
$$

Then we obtain:

$$
\begin{aligned}
e_{n+1}= & X_{n}-X\left(t_{n}\right)+\frac{h}{2}\left\{f\left(X_{n}, t_{n}\right)-f\left(X\left(t_{\xi}\right), t_{\xi}\right)\right\} \\
& +\frac{h}{2}\left\{f\left(X_{n}+f\left(X_{n}, t_{n}\right), t_{n+1}\right)-f\left(X\left(t_{\xi}\right), t_{\xi}\right)\right\}
\end{aligned}
$$

By taking the norm for the two sides:

$$
\begin{aligned}
\left\|e_{n+1}\right\|= & \| X_{n}-X\left(t_{n}\right)+\frac{h}{2}\left\{f\left(X_{n}, t_{n}\right)-f\left(X\left(t_{\xi}\right), t_{\xi}\right)\right\} \\
& +\frac{h}{2}\left\{f\left(X_{n}+f\left(X_{n}, t_{n}\right), t_{n+1}\right)-f\left(X\left(t_{\xi}\right), t_{\xi}\right)\right\}\|\| \\
\leq & \left\|X_{n}-X\left(t_{n}\right)\right\|+\frac{h}{2}\left\|f\left(X\left(t_{\xi}\right), t_{\xi}\right)-f\left(X_{n}, t_{n}\right)\right\| \\
& +\frac{h}{2}\left\|f\left(X_{n}+f\left(X_{n}, t_{n}\right), t_{n+1}\right)-f\left(X\left(t_{\xi}\right), t_{\xi}\right)\right\|
\end{aligned}
$$


Since:

$$
\begin{aligned}
\| & f\left(X\left(t_{\xi}\right), t_{\xi}\right)-f\left(X_{n}, t_{n}\right) \| \\
= & \| f\left(X\left(t_{\xi}\right), t_{\xi}\right)-f\left(X\left(t_{\xi}\right), t_{n}\right)+f\left(X\left(t_{\xi}\right), t_{n}\right) \\
& +f\left(X\left(t_{n}\right), t_{n}\right)-f\left(X\left(t_{n}\right), t_{n}\right)-f\left(X_{n}, t_{n}\right) \| \\
\leq & \left\|f\left(X\left(t_{\xi}\right), t_{\xi}\right)-f\left(X\left(t_{\xi}\right), t_{n}\right)\right\|+\left\|f\left(X\left(t_{\xi}\right), t_{n}\right)-f\left(X\left(t_{n}\right), t_{n}\right)\right\| \\
& +\left\|f\left(X\left(t_{n}\right), t_{n}\right)-f\left(X_{n}, t_{n}\right)\right\|
\end{aligned}
$$

Since the theoretical solution $X$ is m.s. bounded in $\left[t_{0}, t_{1}\right], \quad \sup \|X(t)\| \leq M<\infty$ and

Under hypothesis $\mathrm{C} 1, \mathrm{C} 2 \mathrm{We}$ have

- $\left\|f\left(X\left(t_{\xi}\right), t_{\xi}\right)-f\left(X\left(t_{\xi}\right), t_{n}\right)\right\|=w(h)$

- $\left\|f\left(X\left(t_{\xi}\right), t_{n}\right)-f\left(X\left(t_{n}\right), t_{n}\right)\right\| \leq k\left(t_{n}\right) M h$

where $k\left(t_{n}\right)$ Is Lipschitz constant (from C2) and:

From Theorem (3.3.1) we have $X(t)-X\left(t_{0}\right)=\dot{X}(\xi)\left(t-t_{0}\right)$ and note that the two points
Are $X\left(t_{\xi}\right)$ and $X\left(t_{n}\right)$ in $(*)$ then

$$
\left\|X\left(t_{\xi}\right)-X\left(t_{n}\right)\right\|=\|\dot{X}(\xi)\|\left|t_{\xi}-t_{n}\right| \leq M h
$$

where $\left|t_{\xi}-t_{n}\right|=h$ and $M=\sup _{t_{0} \leq t \leq t_{1}}\|\dot{X}(t)\|$

- $\left\|f\left(X\left(t_{n}\right), t_{n}\right)-f\left(X_{n}, t_{n}\right)\right\|$ $\leq k\left(t_{n}\right)\left\|X\left(t_{n}\right)-X_{n}\right\|=k\left(t_{n}\right)\left\|e_{n}\right\|$

Then by substituting in (3.50) we have

$$
\left\|f\left(X\left(t_{\xi}\right), t_{\xi}\right)-f\left(X_{n}, t_{n}\right)\right\| \leq w(h)+k\left(t_{n}\right) M h+k\left(t_{n}\right)\left\|e_{n}\right\|
$$

And another term:

$$
\begin{aligned}
& \left\|f\left(X_{n}+f\left(X_{n}, t_{n}\right), t_{n+1}\right)-f\left(X\left(t_{\xi}\right), t_{\xi}\right)\right\| \\
= & \left\|f\left(X\left(t_{\xi}\right), t_{\xi}\right)-f\left(X_{n}+f\left(X_{n}, t_{n}\right), t_{n+1}\right)\right\| \\
= & \| f\left(X\left(t_{\xi}\right), t_{\xi}\right)-f\left(X\left(t_{\xi}\right), t_{n+1}\right)+f\left(X\left(t_{\xi}\right), t_{n+1}\right) \\
& +f\left(X\left(t_{n}\right), t_{n+1}\right)-f\left(X\left(t_{n}\right), t_{n+1}\right)-f\left(X_{n}+f\left(X_{n}, t_{n}\right), t_{n+1}\right) \| \\
\leq & \left\|f\left(X\left(t_{\xi}\right), t_{\xi}\right)-f\left(X\left(t_{\xi}\right), t_{n+1}\right)\right\|+\left\|f\left(X\left(t_{\xi}\right), t_{n+1}\right)-f\left(X\left(t_{n}\right), t_{n+1}\right)\right\| \\
& +\left\|f\left(X\left(t_{n}\right), t_{n+1}\right)-f\left(X_{n}+f\left(X_{n}, t_{n}\right), t_{n+1}\right)\right\| \\
\leq & w(h)+k\left(t_{n+1}\right) M h+k\left(t_{n+1}\right)\left[\left\|e_{n}\right\|-M\right]
\end{aligned}
$$

Since:

- $\left\|f\left(X\left(t_{\xi}\right), t_{\xi}\right)-f\left(X\left(t_{\xi}\right), t_{n+1}\right)\right\| \leq w(h)$

- $\left\|f\left(X\left(t_{\xi}\right), t_{n+1}\right)-f\left(X\left(t_{n}\right), t_{n+1}\right)\right\| \leq k\left(t_{n+1}\right) M h$

where $k\left(t_{n}\right)$. Is Lipschitz constant (from C2) and:

From Theorem (3.3.1) we have

$X(t)-X\left(t_{0}\right)=\dot{X}(\xi)\left(t-t_{0}\right)$ and note that the two points are $X\left(t_{\xi}\right)$ and $X\left(t_{n}\right)$ in (*) then we have:

$$
\left\|X\left(t_{\xi}\right)-X\left(t_{n}\right)\right\|=\|\dot{X}(\xi)\|\left|t_{\xi}-t_{n}\right| \leq M h
$$

where $\left|t_{\xi}-t_{n}\right|=h$ and $M=\sup _{t_{0} \leq t \leq t_{1}}\|\dot{X}(t)\|$

And the last term:

$$
\begin{aligned}
& \left\|f\left(X\left(t_{n}\right), t_{n+1}\right)-f\left(X_{n}+f\left(X_{n}, t_{n}\right), t_{n+1}\right)\right\| \\
& \leq k\left(t_{n+1}\right)\left\|X\left(t_{n}\right)-X_{n}-f\left(X_{n}, t_{n}\right)\right\| \\
& \leq k\left(t_{n+1}\right)\left\|X\left(t_{n}\right)-X_{n}\right\|-\left\|f\left(X_{n}, t_{n}\right)\right\| \\
& =k\left(t_{n+1}\right)\left[\left\|e_{n}\right\|-M\right]
\end{aligned}
$$

Then by substituting in (3.49) we have 


$$
\begin{aligned}
& \left\|e_{n+1}\right\| \leq\left\|e_{n}\right\|+\frac{h}{2}\left[w(h)+k\left(t_{n}\right) M h+k\left(t_{n}\right)\left\|e_{n}\right\|\right]+\frac{h}{2}\left[w(h)+k\left(t_{n+1}\right) M h+k\left(t_{n+1}\right)\left[\left\|e_{n}\right\|-M\right]\right] \\
& =\left\|e_{n}\right\|\left(1+\frac{h}{2} k\left(t_{n}\right)+k\left(t_{n+1}\right)\right)+\frac{h}{2}\left[2 w(h)+h k\left(t_{n}\right) M+h k\left(t_{n+1}\right) M-K\left(t_{n+1}\right) M\right] \\
& \leq\left\{\left\|e_{n-1}\right\|\left(1+\frac{h}{2} k\left(t_{n}\right)+k\left(t_{n+1}\right)\right)+\frac{h}{2}\left[2 w(h)+h k\left(t_{n}\right) M+h k\left(t_{n+1}\right) M-K\left(t_{n}\right) M\right]\right\} \\
& \left\{\left(1+\frac{h}{2} k\left(t_{n}\right)+k\left(t_{n+1}\right)\right)\right\}+\frac{h}{2}\left[2 w(h)+h k\left(t_{n}\right) M+h k\left(t_{n+1}\right) M-K\left(t_{n+1}\right) M\right] \\
& =\left\|e_{n-1}\right\|\left(1+\frac{h}{2} k\left(t_{n}\right)+k\left(t_{n+1}\right)\right)^{2}+\frac{h}{2}\left[2 w(h)+h k\left(t_{n}\right) M+h k\left(t_{n+1}\right) M-K\left(t_{n+1}\right) M\right]\left(2+\frac{h}{2} k\left(t_{n}\right)+k\left(t_{n+1}\right)\right) \\
& \leq\left\{\left\|e_{n-2}\right\|\left(1+\frac{h}{2} k\left(t_{n}\right)+k\left(t_{n+1}\right)\right)+\frac{h}{2}\left[2 w(h)+h k\left(t_{n}\right) M+h k\left(t_{n+1}\right) M-K\left(t_{n}\right) M\right]\right\} \\
& \left\{\left(1+\frac{h}{2} k\left(t_{n}\right)+k\left(t_{n+1}\right)\right)^{2}\right\}+\frac{h}{2}\left[2 w(h)+h k\left(t_{n}\right) M+h k\left(t_{n+1}\right) M-K\left(t_{n+1}\right) M\right]\left(2+\frac{h}{2} k\left(t_{n}\right)+k\left(t_{n+1}\right)\right) \\
& =\left\|e_{n-2}\right\|\left(1+\frac{h}{2} k\left(t_{n}\right)+k\left(t_{n+1}\right)\right)^{3}+\frac{h}{2}\left[2 w(h)+h k\left(t_{n}\right) M+h k\left(t_{n+1}\right) M-K\left(t_{n+1}\right) M\right] \\
& {\left[1+\left(1+\frac{h}{2} k\left(t_{n}\right)+k\left(t_{n+1}\right)\right)+\left(1+\frac{h}{2} k\left(t_{n}\right)+k\left(t_{n+1}\right)\right)^{2}\right]}
\end{aligned}
$$

Then we have:

$$
\begin{aligned}
& \left\|e_{n+1}\right\| \leq\left\|e_{0}\right\|\left(1+\frac{h}{2} k\left(t_{n}\right)+k\left(t_{n+1}\right)\right)^{n+1}+\frac{h}{2}\left[2 w(h)+h k\left(t_{n}\right) M+h k\left(t_{n+1}\right) M-K\left(t_{n+1}\right) M\right] \\
& {\left[1+\left(1+\frac{h}{2} k\left(t_{n}\right)+k\left(t_{n+1}\right)\right)+\left(1+\frac{h}{2} k\left(t_{n}\right)+k\left(t_{n+1}\right)\right)^{2}+\cdots+\left(1+\frac{h}{2} k\left(t_{n}\right)+k\left(t_{n+1}\right)\right)^{n}\right]}
\end{aligned}
$$

Since:

$$
\left[1+\left(1+\frac{h}{2} k\left(t_{n}\right)+k\left(t_{n+1}\right)\right)+\left(1+\frac{h}{2} k\left(t_{n}\right)+k\left(t_{n+1}\right)\right)^{2}+\cdots+\left(1+\frac{h}{2} k\left(t_{n}\right)+k\left(t_{n+1}\right)\right)^{n}\right]
$$

is geometrical sequence then we have:

$$
\left[1+\left(1+\frac{h}{2} k\left(t_{n}\right)+k\left(t_{n+1}\right)\right)+\left(1+\frac{h}{2} k\left(t_{n}\right)+k\left(t_{n+1}\right)\right)^{2}+\cdots+\left(1+\frac{h}{2} k\left(t_{n}\right)+k\left(t_{n+1}\right)\right)^{n}\right]=\frac{\left[\left(1+\frac{h}{2} k\left(t_{n}\right)+k\left(t_{n+1}\right)\right)^{n}\right]-1}{\frac{h}{2} k\left(t_{n}\right)+k\left(t_{n+1}\right)}
$$

Then we get:

$$
\left\|e_{n+1}\right\| \leq\left\|e_{0}\right\|\left(1+\frac{h}{2} k\left(t_{n}\right)+k\left(t_{n+1}\right)\right)^{n+1}+\frac{h}{2}\left[2 w(h)+h k\left(t_{n}\right) M+h k\left(t_{n+1}\right) M-K\left(t_{n+1}\right) M\right] \frac{\left[\left(1+\frac{h}{2} k\left(t_{n}\right)+k\left(t_{n+1}\right)\right)^{n}\right]-1}{\frac{h}{2} k\left(t_{n}\right)+k\left(t_{n+1}\right)}
$$

Taking into account that $e_{0}=0$ where

$$
e_{0}=X_{0}-X\left(t_{0}\right)=0
$$




$$
\begin{gathered}
\left\|e_{n+1}\right\| \leq \frac{h}{2}\left[2 w(h)+h k\left(t_{n}\right) M+h k\left(t_{n+1}\right) M-k\left(t_{n+1}\right) M\right] \frac{\left[\left(1+\frac{h}{2} k\left(t_{n}\right)+k\left(t_{n+1}\right)\right)^{n}\right]-1}{\frac{h}{2} k\left(t_{n}\right)+k\left(t_{n+1}\right)} \\
\lim _{h \rightarrow 0}\left\|e_{n+1}\right\| \leq \lim _{h \rightarrow 0} \frac{h}{2}\left[2 w(h)+k\left(t_{n}\right) M h+h k\left(t_{n+1}\right) M-K\left(t_{n+1}\right) M\right] \frac{\left[\left(1+\frac{h}{2} k\left(t_{n}\right)+k\left(t_{n+1}\right)\right)^{n}\right]-1}{\frac{h}{2} k\left(t_{n}\right)+k\left(t_{n+1}\right)} \\
=\lim _{h \rightarrow 0} \frac{\frac{h}{2}\left[2 w(h)+k\left(t_{n}\right) M h+k\left(t_{n+1}\right) M h-k\left(t_{n+1}\right) M\right]}{\frac{h}{2} k\left(t_{n}\right)+k\left(t_{n+1}\right)}\left[\left(1+\frac{h}{2} K\left(t_{n}\right)+k\left(t_{n+1}\right)\right)^{n}\right] \\
-\lim _{h \rightarrow 0} \frac{\frac{h}{2}\left[2 w(h)+k\left(t_{n}\right) M h+k\left(t_{n+1}\right) M h-k\left(t_{n+1}\right) M\right]}{\frac{h}{2} k\left(t_{n}\right)+K\left(t_{n+1}\right)}
\end{gathered}
$$

Note that:

The term:

and the second term:

$$
\lim _{h \rightarrow 0} \frac{\frac{h}{2}\left[2 w(h)+k\left(t_{n}\right) M h+k\left(t_{n+1}\right) M h-k\left(t_{n+1}\right) M\right]}{\frac{h}{2} k\left(t_{n}\right)+K\left(t_{n+1}\right)}=\frac{0}{k\left(t_{n+1}\right)}=0
$$

$$
\lim _{h \rightarrow 0} \frac{\frac{h}{2}\left[2 w(h)+k\left(t_{n}\right) M h+k\left(t_{n+1}\right) M h-k\left(t_{n+1}\right) M\right]}{\frac{h}{2} k\left(t_{n}\right)+k\left(t_{n+1}\right)}\left[\left(1+\frac{h}{2} K\left(t_{n}\right)+k\left(t_{n+1}\right)\right)^{n}\right]
$$

we have:

$$
\begin{aligned}
& \lim _{h \rightarrow 0} \frac{\frac{h}{2}\left[2 w(h)+k\left(t_{n}\right) M h+k\left(t_{n+1}\right) M h-k\left(t_{n+1}\right) M\right]}{\frac{h}{2} k\left(t_{n}\right)+k\left(t_{n+1}\right)}\left[\left(1+\frac{h}{2} K\left(t_{n}\right)+k\left(t_{n+1}\right)\right)^{n}\right] \\
& =\lim _{h \rightarrow 0} \frac{\frac{h}{2}\left[2 w(h)+k\left(t_{n}\right) M h+k\left(t_{n+1}\right) M h-k\left(t_{n+1}\right) M\right]}{\frac{h}{2} k\left(t_{n}\right)+k\left(t_{n+1}\right)} \lim _{h \rightarrow 0}\left[\left(1+\frac{h}{2} K\left(t_{n}\right)+k\left(t_{n+1}\right)\right)^{n}\right]
\end{aligned}
$$

The first limit in (3.53) equals zero and:

The computation of

$$
\begin{aligned}
& \lim _{h \rightarrow 0}\left[\left(1+\frac{h}{2} K\left(t_{n}\right)+k\left(t_{n+1}\right)\right)^{n}\right] \text { is as follows: } \\
& \text { Let } y=\lim _{h \rightarrow 0}\left[\left(1+\frac{h}{2} K\left(t_{n}\right)+k\left(t_{n+1}\right)\right)^{n}\right] \text { then by tack- } \\
& \ln y=\ln \lim _{h \rightarrow 0}\left[\left(1+\frac{h}{2} K\left(t_{n}\right)+k\left(t_{n+1}\right)\right)^{n}\right]=\lim _{h \rightarrow 0} \ln \left[\left(1+\frac{h}{2} K\left(t_{n}\right)+k\left(t_{n+1}\right)\right)^{n}\right] \\
& =\lim _{h \rightarrow 0}\left\{\left\{\ln \left[\left(1+\frac{h}{2} K\left(t_{n}\right)+k\left(t_{n+1}\right)\right)\right]\right\}=\lim _{h \rightarrow 0} \frac{t_{n}-t_{0}}{h}\left[\ln \left[\left(1+\frac{h}{2} K\left(t_{n}\right)+k\left(t_{n+1}\right)\right)\right]\right.\right. \\
& =\lim _{h \rightarrow 0} \frac{\left(t_{n}-t_{0}\right)\left[\ln \left(1+\frac{h}{2} k\left(t_{n}\right)+k\left(t_{n+1}\right)\right]\right.}{h}
\end{aligned}
$$


By using the (L'Hospital's Rule):

$\lim _{h \rightarrow 0} \frac{\left(t_{n}-t_{0}\right)\left[\ln \left(1+\frac{h}{2} k\left(t_{n}\right)+k\left(t_{n+1}\right)\right)\right]}{h}$
$=\lim _{h \rightarrow 0} \frac{\left(t_{n}-t_{0}\right) \frac{1}{\left(1+\frac{h}{2} k\left(t_{n}\right)+k\left(t_{n+1}\right)\right)}\left[\frac{1}{2} k\left(t_{n}\right)\right]}{1}$

$=\lim _{h \rightarrow 0} \frac{\frac{1}{2}\left(t_{n}-t_{0}\right) k\left(t_{n}\right)}{1+\frac{h}{2} k\left(t_{n}\right)+k\left(t_{n+1}\right)}=\frac{\frac{1}{2}\left(t-t_{0}\right) k(t)}{1+k(t)}$

$\ln y=\frac{\left(t-t_{0}\right) k(t)}{2[1+k(t)]}$ Then $y=\mathrm{e}^{\frac{\left(t-t_{0}\right) k(t)}{2[1+k(t)]}}$ hence:

$$
\lim _{h \rightarrow 0}\left[\left(1+\frac{h}{2} K\left(t_{n}\right) h+k\left(t_{n+1}\right)\right)^{n}\right]=\mathrm{e}^{\frac{\left(t-t_{0}\right) k(t)}{2[1+k(t)]}}
$$

By substituting in (3.53):

$$
\begin{aligned}
& \lim _{h \rightarrow 0} \frac{\frac{h}{2}\left[2 w(h)+k\left(t_{n}\right) M h+k\left(t_{n+1}\right) M h-k\left(t_{n+1}\right) M\right]}{\frac{h}{2} k\left(t_{n}\right)+k\left(t_{n+1}\right)} \\
& {\left[\left(1+\frac{h}{2} K\left(t_{n}\right)+k\left(t_{n+1}\right)\right)^{n}\right]=0 \times e^{\frac{\left(t-t_{0}\right) k(t)}{2[1+k(t)]}}=0}
\end{aligned}
$$

By substituting from (3.55) and (3.53) in (3.51) then we obtain $\lim _{h \rightarrow 0}\left\|e_{n+1}\right\| \rightarrow 0$ i.e. $\left\{e_{n}\right\}$ converges in $\mathrm{m}$.s to zero as $h \rightarrow 0$ hence $X_{n} \stackrel{\text { m.s }}{\longrightarrow} X\left(t_{n}\right)=X(t)$

\section{Some Results}

\section{Theorem 4.1}

Let $\left\{X_{n}, n=0,1, \cdots\right\},\left\{Y_{n}, n=0,1, \cdots\right\}$ be sequences of 2-r.v's over the same probability space and let $a$ and $b$ be deterministic real numbers.

Suppose: $X=\lim _{n \rightarrow \infty} X_{n}$ and $Y=\lim _{n \rightarrow \infty} Y_{n}$

Then:
1) $(a X+b Y)=\lim _{n \rightarrow \infty}\left(a X_{n}+b Y_{n}\right)$
2) $E(X)=\lim _{n \rightarrow \infty} E\left\{X_{n}\right\}$
3) $E(X Y)=\lim _{n \rightarrow \infty} E\left\{X_{n} Y_{n}\right\}$
4) $\lim _{n \rightarrow \infty} E\left(X_{n}^{2}\right)=E\left(X^{2}\right)$
5) $\lim _{n \rightarrow \infty} \operatorname{Var}\left(X_{n}\right)==\operatorname{Var}(X)$

Definition 4.1 [13]. "The convergence in probability"
A sequence of r.v's $\left\{X_{n}\right\}$ converges in probability to a random variable $X$ as $n \rightarrow \infty$ if

$$
\lim _{n \rightarrow \infty} p\left\{\left|X_{n}-X\right|>\varepsilon\right\}=0 \forall \varepsilon>0
$$

\section{Definition 4.2 [13]. "The convergence in distribu-} tion”

A sequence of r.v's $\left\{X_{n}\right\}$ converge in distribution to a random variable $X$ as $n \rightarrow \infty$ if

$$
\lim _{n \rightarrow \infty} F_{x_{n}}(x)=F_{x}(x)
$$

Lemma (4.1) [13]

The convergence in m.s implies convergence in probability

\section{Lemma (4.2) [13]}

The convergence in probability implies convergence in distribution

\section{Theorem 4.2}

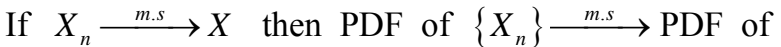
$\{X\}$ i.e.; $\lim _{n \rightarrow \infty} f_{x_{n}}(x)=f_{X}(x)$

Proof

Since we have shown that If $X_{n} \stackrel{\text { m.s }}{\longrightarrow} X$ then $X_{n} \stackrel{d}{\longrightarrow} X$

i.e., if $X_{n} \stackrel{m . s}{\longrightarrow} X$ then $\lim F_{X_{n}}(x)=F_{X}(x)$

Then we have: $\lim _{n \rightarrow \infty} \frac{\mathrm{d}}{\mathrm{d} x} F_{X_{n}}(x)=\frac{\mathrm{d}}{\mathrm{d} x} F_{X}(x)$ then

$\lim _{n \rightarrow \infty} f_{x_{n}}(x)=f_{X}(x)$

\section{Numerical Examples}

\section{Example (5.1)}

The differential equation with random term in it and random initial condition

$$
y^{\prime}=K x, y\left(x_{0}\right)=D, x \in\left[x_{0}, x_{n}\right],
$$

$K, D$ are independent Poisson random variables with joint PDF

$$
f_{K, D}(K, D)=\frac{\mathrm{e}^{-4} 2^{k+D}}{k ! D !}, K, D=0,1,2, \cdots
$$

\section{1) The exact solution,}

$$
y=D+\frac{K\left(x^{2}-x_{0}^{2}\right)}{2}
$$

\section{2) The numerical solution}

Using the Random Euler Method:

$$
y_{n}=y_{n-1}+h f\left(y_{n-1}, x_{n-1}\right), y\left(x_{0}\right)=y_{0}
$$

at $n=1$

$$
y_{1}=y_{0}+h f\left(y_{0}, x_{0}\right)=D+h K x_{0}
$$

at $n=2$ 


$$
\begin{aligned}
y_{2} & =y_{1}+h f\left(y_{1}, x_{1}\right)=D+h K x_{0}+h K x_{1} \\
& =D+h K x_{0}+h K\left(x_{0}+h\right)
\end{aligned}
$$

at $n=3$

$$
\begin{aligned}
y_{3} & =y_{2}+h f\left(y_{2}, x_{2}\right)=D+h K x_{0}+h K x_{1}+h K x_{2} \\
& =D+h K x_{0}+h K\left(x_{0}+h\right)+h K\left(x_{0}+2 h\right)
\end{aligned}
$$

at $n=4$

$$
\begin{aligned}
y_{4} & =y_{3}+h f\left(y_{3}, x_{3}\right)=D+h K x_{0}+h K x_{1}+h K x_{2}+h K x_{3} \\
& =D+h K x_{0}+h K\left(x_{0}+h\right)+h K\left(x_{0}+2 h\right)+h K\left(x_{0}+3 h\right)
\end{aligned}
$$

and so on...

Then the general numerical solution is

$$
\begin{aligned}
y_{n}= & D+h K x_{0}+h K\left(x_{0}+h\right)+h K\left(x_{0}+2 h\right) \\
& +h K\left(x_{0}+3 h\right)+\cdots+h K\left(x_{0}+(n-1) h\right)
\end{aligned}
$$

i.e., $y_{n}=D+h K \sum_{i=0}^{n-1}\left(x_{0}+i h\right)$.

This can be written in another form:

$$
\begin{aligned}
\left|y_{n}-y\right|^{2}= & K^{2} x_{0}^{2}\left(x_{n}-x_{0}\right)^{2}+K^{2} x_{0}\left(x_{n}-x_{0}\right)\left[\frac{(n-1)}{n}\left(x_{n}-x_{0}\right)^{2}-\left(x^{2}-x_{0}^{2}\right)\right] \\
& +\frac{K^{2}}{4}\left[\frac{(n-1)}{n}\left(x_{n}-x_{0}\right)^{2}-\left(x^{2}-x_{0}^{2}\right)\right]^{2} \\
= & K^{2} x_{0}^{2} x_{n}^{2}-2 K^{2} x_{n} x_{0}^{3}+K^{2} x_{0}^{4}+K^{2} x_{n} x_{0}\left(\frac{n-1}{n}\right)\left(x_{n}-x_{0}\right)^{2}-K^{2} x_{0}^{2}\left(\frac{n-1}{n}\right)\left(x_{n}-x_{0}\right)^{2} \\
& -K^{2} x_{n} x_{0}\left(x^{2}-x_{0}^{2}\right)+K^{2} x_{0}^{2}\left(x^{2}-x_{0}^{2}\right)+\frac{K^{2}}{4}\left(\frac{n-1}{n}\right)^{2}\left(x_{n}-x_{0}\right)^{4} \\
& -\frac{K^{2}}{2}\left(\frac{n-1}{n}\right)\left(x_{n}-x_{0}\right)^{2}\left(x^{2}-x_{0}^{2}\right)+\frac{K^{2}}{4}\left(x^{2}-x_{0}^{2}\right)^{2} \\
& \lim _{n \rightarrow \infty} E\left|y_{n}-y\right|^{2}=18 x_{0}^{2} x^{2}-36 x_{0}^{3} x+18 x_{0}^{4}+18 x x_{0}\left(x-x_{0}\right)^{2}-18 x_{0}^{2}\left(x-x_{0}\right)^{2} \\
& -18 x x_{0}\left(x^{2}-x_{0}^{2}\right)+18 x_{0}^{2}\left(x^{2}-x_{0}^{2}\right)+\frac{18}{4} x^{4}-18 x^{3} x_{0}+27 x^{2} x_{0}^{2}-18 x x_{0}^{3} \\
& +\frac{18}{4} x^{4}-9 x^{2} x_{0}^{2}+\frac{18}{4} x_{0}^{4}=0 \\
& +27 x^{2} x_{0}^{2}-9 x^{4}+18 x^{3} x_{0}-18 x x_{0}^{3}+9 x_{0}^{4}+\frac{18}{4} x^{4}-9 x^{2} x_{0}^{2}+\frac{18}{4} x_{0}^{4} \\
= & 18 x_{0}^{2} x^{2}-36 x_{0}^{3} x+18 x_{0}^{4}+72 x x_{0}^{3}-36 x^{2} x_{0}^{2}-36 x_{0}^{4}+\frac{18}{4} x^{4}-18 x^{3} x_{0} \\
& \\
& \\
&
\end{aligned}
$$

i.e; $\lim _{n \rightarrow \infty} y_{n}=y$
We can verify theorem (4.1) as follows 
2) $\lim _{n \rightarrow \infty} E\left\{y_{n}\right\}=E\{y\}$

Proof

$$
\begin{aligned}
y_{n}= & D+h K \sum_{i=0}^{n-1}\left(x_{0}+i h\right)=D+h K \sum_{j=1}^{n}\left(x_{0}+(j-1) h\right)=D+h K\left[x_{0}+\left(x_{0}+h\right)+\left(x_{0}+2 h\right)+\left(x_{0}+3 h\right)+\cdots\left(x_{0}+(n-1) h\right)\right] \\
& \left.=D+h K\left[n x_{0}+h[1+2+3+\cdots+(n-1)]\right]=D+h K\left[n x_{0}+h\left(\frac{n(n-1)}{2}\right)\right]\right] \\
& =D+K\left[n h x_{0}+h^{2}\left(\frac{n(n-1)}{2}\right)\right] \text { where } h=\frac{x_{n}-x_{0}}{n} \\
& =D+K\left[n x_{0}+\frac{\left(x_{n}-x_{0}\right)}{n}+\left(\frac{\left(x_{n}-x_{0}\right)}{n}\right)^{2}\left(\frac{n(n-1)}{2}\right)\right]=D+K\left[x_{0}\left(x_{n}-x_{0}\right)+\frac{(n-1)}{2 n}\left(x_{n}-x_{0}\right)^{2}\right] \\
& E\left(y_{n}\right)=E(D)+E\left\{K\left[x_{0}\left(x_{n}-x_{0}\right)+\frac{(n-1)}{2 n}\left(x_{n}-x_{0}\right)^{2}\right]\right\}=2+2\left\{E\left(x_{0} x_{n}\right)-E\left(x_{0}^{2}\right)+\frac{(n-1)}{2 n} E\left(x_{n}-x_{0}\right)^{2}\right\}
\end{aligned}
$$

Then:

$$
\begin{aligned}
\lim _{n \rightarrow \infty} E\left\{y_{n}\right\} & =2+2\left\{E\left(x_{0} x\right)-E\left(x_{0}^{2}\right)+\frac{1}{2} E\left(x-x_{0}\right)^{2}\right\} \\
& =2+2\left\{E\left(x_{0} x\right)-E\left(x_{0}^{2}\right)+\frac{1}{2} E\left(x^{2}\right)-E\left(x_{0} x\right)+\frac{1}{2} E\left(x_{0}^{2}\right)\right\} \\
& =2+2\left\{\frac{1}{2} E\left(x^{2}\right)-\frac{1}{2} E\left(x_{0}^{2}\right)\right\}=2+\left\{E\left(x^{2}\right)-E\left(x_{0}^{2}\right)\right\}=E\{y\}
\end{aligned}
$$

i.e.; $\lim _{n \rightarrow \infty} E\left\{y_{n}\right\}=E\{y\}$.

3) $\lim _{n \rightarrow \infty} E\left\{y_{n}^{2}\right\}=E\left\{y^{2}\right\}$

Proof
Since $y_{n}=D+n h K x_{0}+[n(n-1) / 2] K h^{2}$

Then we have:

$$
\begin{aligned}
& E\left\{y_{n}^{2}\right\}=E\left[D+K x_{0}\left(x_{n}-x_{0}\right)+K n(n-1) / 2\right]\left[\left(x_{n}-x_{0}\right)^{2} / n^{2}\right]^{2} \\
&=E\left[D+K\left(x_{n}-x_{0}\right) x_{0}\right]^{2}+2 E\left\{\left[D+K\left(x_{n}-x_{0}\right) x_{0}\right]\left[K[(n-1) / n]\left[\left(x_{n}-x_{0}\right)^{2} / 2\right]\right]\right\} \\
&+ E\left[K[n(n-1) / n]\left[\left(x_{n}-x_{0}\right)^{2} / 2\right]\right]^{2} \\
&= E\left[D^{2}\right]+E\left[K\left(x_{n}-x_{0}\right) x_{0}\right]^{2}+2 E\left[D K\left(x_{n} x_{0}-x_{0}^{2}\right)\right]+2 E\left\{\left[D+K\left(x_{n}-x_{0}\right) x_{0}\right]\left[K[(n-1) / n]\left[\left(x_{n}-x_{0}\right)^{2} / 2\right]\right]\right\} \\
&+E\left[K^{2}[n(n-1) / n]^{2} E\left[\left(x_{n}-x_{0}\right)^{2} / 2\right]^{2}\right. \\
&=E {\left[D^{2}\right]+E\left[K^{2}\right] E\left[x_{n} x_{0}\right]^{2}-2 E\left[K^{2}\right] E\left[x_{n} x_{0}^{3}\right]+E\left[K^{2}\right] E\left[x_{0}\right]^{4}+2 E[D] E[K] E\left[x_{n} x_{0}\right]-2 E[D] E[K] E\left[x_{0}^{2}\right] } \\
&+2 E[D] E[K][(n-1) / n] E\left[\left(x_{n}-x_{0}\right)^{2} / 2\right]+2[(n-1) / n] E\left[K^{2}\right] E\left[x_{0}\left(x-x_{0}\right)^{3} / 2\right] \\
&+[n(n-1) / n]^{2} E\left[K^{2}\right] E\left[\left(x_{n}-x_{0}\right)^{2} / 2\right]^{2} \\
&= E[D]^{2}+E\left[K^{2}\right] E\left[x_{n} x_{0}\right]^{2}-2 E\left[K^{2}\right] E\left[x_{n} x_{0}^{3}\right]+E\left[K^{2}\right] E\left[x_{0}\right]^{4}+2 E[D] E[K] E\left[x_{n} x_{0}\right]-2 E[D] E[K] E\left[x_{0}^{2}\right] \\
&+E[D] E[K][(n-1) / n] E\left[\left(x_{n}-x_{0}\right)^{2}\right]+[(n-1) / n] E\left[K^{2}\right] E\left[x_{0}\left(x-x_{0}\right)^{3}\right]+\frac{1}{4}[n(n-1) / n]^{2} E\left[K^{2}\right] E\left[\left(x_{n}-x_{0}\right)^{4}\right]
\end{aligned}
$$


Then by taking the limit:

$$
\begin{aligned}
\lim _{n \rightarrow \infty} E\left\{y_{n}^{2}\right\}= & 6+6 E\left[x x_{0}\right]^{2}-12 E\left[x x_{0}^{3}\right]+6 E\left[x_{0}\right]^{4}+8 E\left[x x_{0}\right]-8 E\left[x_{0}^{2}\right] \\
& +4 E\left(x-x_{0}\right)^{2}+6 E\left[x_{0}\left(x-x_{0}\right)^{3}\right]+\frac{6}{4} E\left(x-x_{0}\right)^{4} \\
= & 6+6 E\left[x x_{0}\right]^{2}-12 E\left[x x_{0}^{3}\right]+6 E\left[x_{0}\right]^{4}+8 E\left[x x_{0}\right]-8 E\left[x_{0}^{2}\right]+4 E\left[x^{2}\right] \\
& -8 E\left[x x_{0}\right]+4 E\left[x_{0}^{2}\right]+6 E\left[x^{3} x_{0}\right]-18 E\left[x^{2} x_{0}^{2}\right]+18 E\left[x x_{0}^{3}\right]-6 E\left[x_{0}^{4}\right] \\
& +\frac{6}{4} E\left[x^{4}\right]-6 E\left[x^{3} x_{0}\right]-6 E\left[x x_{0}^{3}\right]+9 E\left[x^{2} x_{0}^{2}\right]+\frac{6}{4} E\left[x_{0}^{4}\right]=E\left(y^{2}\right)
\end{aligned}
$$

i.e. $\lim _{n \rightarrow \infty} E\left\{y_{n}^{2}\right\}=E\left\{y^{2}\right\}$.

4) $\lim _{n \rightarrow \infty} \operatorname{var}\left\{y_{n}\right\}=\operatorname{var}\{y\}$

Proof

$$
\begin{aligned}
\lim _{n \rightarrow \infty} \operatorname{Var}\left\{y_{n}\right\} & =\lim _{n \rightarrow \infty}\left[E\left(y_{n}^{2}\right)-\left[E\left(y_{n}\right)\right]^{2}\right] \\
& =\lim _{n \rightarrow \infty} E\left(y_{n}^{2}\right)-\lim _{n \rightarrow \infty}\left[E\left(y_{n}\right)\right]^{2} \\
& =E\left(y^{2}\right)-[E(y)]^{2}=\operatorname{Var}(y)
\end{aligned}
$$

i.e., $\lim \operatorname{Var}\left(y_{n}\right)=\operatorname{Var}(y)$.

5) $\lim _{n \rightarrow \infty} \operatorname{PDF}\left(y_{n}\right)=\operatorname{PDF}(y)$

Proof

Since $y=D+\frac{K\left(x^{2}-x_{0}^{2}\right)}{2}$

Let us define $Z=D$. Then the inverse transformation is:

$D=Y-\frac{K\left(x^{2}-x_{0}^{2}\right)}{2}, D=Z$ then we have $D=Z$ and $K=\frac{2(y-Z)}{x^{2}-x_{0}^{2}}$

$$
J=\left|\begin{array}{ll}
\frac{\partial k}{\partial y} & \frac{\partial k}{\partial z} \\
\frac{\partial D}{\partial y} & \frac{\partial D}{\partial z}
\end{array}\right|=\left|\begin{array}{cc}
\frac{2}{x^{2}-x_{0}^{2}} & \frac{-2}{x^{2}-x_{0}^{2}} \\
0 & 1
\end{array}\right|=\frac{2}{x^{2}-x_{0}^{2}}
$$

Then:

$$
f_{y, z}(y, z)=f_{K, D}\left(\frac{2(y-Z)}{x^{2}-x_{0}^{2}}, Z\right)|J|=\frac{\mathrm{e}^{-4} 2^{Z+\frac{2(Y-Z)}{X^{2}-X_{0}^{2}}}}{Z !\left[\frac{2(y-Z)}{x^{2}-x_{0}^{2}}\right] !}
$$

Since $D \geq 0$ then $Z \geq 0$ hence

$$
y \geq Z+\frac{K\left(x^{2}-x_{0}^{2}\right)}{2}
$$

$$
f_{y}(y)=\sum_{Z=0}^{y} \frac{\mathrm{e}^{-4} 2^{Z+\frac{2(Y-Z)}{x^{2}-x_{0}^{2}}}}{Z !\left[\frac{2(y-Z)}{x^{2}-x_{0}^{2}}\right] !}=P D F(y) .
$$

For a numerical solution:

$$
\begin{aligned}
& \text { since } y_{n}=D+n h K x_{0}+[n(n-1) / 2] K h^{2} \\
& \text { Let } z_{n}=D \text { then } K=\frac{\left(y_{n}-z_{n}\right)}{n h x_{0}+[n(n-1) / 2] h^{2}} \\
& J=\left|\begin{array}{ll}
\frac{\partial K}{\partial y_{n}} & \frac{\partial K}{\partial Z_{n}} \\
\frac{\partial D}{\partial y_{n}} & \frac{\partial D}{\partial Z_{n}}
\end{array}\right| \\
& =\left|\begin{array}{cc}
\frac{1}{n h x_{0}+[n(n-1) / 2] h^{2}} & \frac{-1}{n h x_{0}+[n(n-1) / 2] h^{2}} \\
0 & 1
\end{array}\right| \\
& =\frac{1}{n h x_{0}+[n(n-1) / 2] h^{2}}
\end{aligned}
$$

Then:

$$
\begin{aligned}
f_{y_{n}, z_{n}}\left(y_{n}, z_{n}\right) & =f_{K, D}\left(\frac{\left(y_{n}-z_{n}\right)}{n h x_{0}+[n(n-1) / 2] h^{2}}, z_{n}\right)|J| \\
& =\frac{\mathrm{e}^{-4} 2^{z_{n}+\frac{\left(Y_{n}-Z_{n}\right)}{n h x_{0}+[n(n-1) / 2] h^{2}}}}{z_{n} !\left[\frac{\left(y_{n}-z_{n}\right)}{n h x_{0}+[n(n-1) / 2] h^{2}}\right] !} \\
f_{y n}\left(y_{n}\right) & =\sum_{z_{n}=0}^{y_{n}} \frac{e^{-4} 2^{Z_{n}+\frac{\left(Y_{n}-z_{n}\right)}{n h x_{0}+[n(n-1) / 2] h^{2}}}}{z_{n} !\left[\frac{\left(y_{n}-z_{n}\right)}{n h x_{0}+[n(n-1) / 2] h^{2}}\right]}
\end{aligned}
$$

where $h=\frac{x_{n}-x_{0}}{n}$ 


$$
\begin{aligned}
f_{y n}\left(y_{n}\right) & =\sum_{z_{n}}^{y_{n}} \frac{\mathrm{e}^{-4} 2^{z_{n}+\frac{\left(Y_{n}-z_{n}\right)}{\left(x_{n}-x_{0}\right) x_{0}+[(n-1) / 2 n]\left(x_{n}-x_{0}\right)^{2}}} z_{n} !\left[\frac{\left(y_{n}-z_{n}\right)}{\left(x_{n}-x_{0}\right) x_{0}+[(n-1) / 2 n]\left(x_{n}-x_{0}\right)^{2}}\right] !}{} \\
& =\operatorname{PDF}\left(y_{n}\right)
\end{aligned}
$$

Then by taking the limit we have

$$
\lim _{n \rightarrow \infty} f_{y_{n}}\left(y_{n}\right)=\sum_{z=0}^{y} \frac{\mathrm{e}^{-4} 2^{Z+\frac{2(Y-z)}{x^{2}-x_{0}^{2}}}}{z !\left[\frac{2(y-z)}{x^{2}-x_{0}^{2}}\right] !}=f_{y}(y)
$$

i.e.; $\lim _{n \rightarrow \infty} P D F\left(y_{n}\right)=P D F(y)$

\section{B. Using the Random Runge-Kutta method:}

$$
y_{n+1}=y_{n}+\frac{h}{2}\left[f\left(y_{n}, x_{n}\right)+f\left(y_{n}+f\left(y_{n}, x_{n}\right), x_{n+1}\right)\right] \text {, }
$$

at $n=0$

$$
\begin{aligned}
y_{1} & =y_{0}+\frac{h}{2}\left[f\left(y_{0}, x_{0}\right)+f\left(y_{0}+f\left(y_{0}, x_{0}\right), x_{1}\right)\right] \\
& =D+\frac{h}{2}\left[K x_{0}+K x_{1}\right]=D+\frac{h K}{2}\left[x_{0}+x_{1}\right] .
\end{aligned}
$$

At $n=1$

$$
\begin{aligned}
y_{2} & =y_{1}+\frac{h}{2}\left[f\left(y_{1}, x_{1}\right)+f\left(y_{1}+f\left(y_{1}, x_{1}\right), x_{2}\right)\right] \\
& =D+\frac{h}{2}\left[K x_{0}+K x_{1}\right]+\frac{h}{2}\left[K x_{1}+K x_{2}\right] \\
& =D+\frac{h K}{2}\left[x_{0}+2 x_{1}+x_{2}\right] .
\end{aligned}
$$

At $n=2$

$$
\begin{aligned}
y_{3} & =y_{2}+\frac{h}{2}\left[f\left(y_{2}, x_{2}\right)+f\left(y_{2}+f\left(y_{2}, x_{2}\right), x_{3}\right)\right] \\
& =D+\frac{h K}{2}\left[x_{0}+2 x_{1}+x_{2}\right]+\frac{h}{2}\left[K x_{2}+K x_{3}\right] \\
& =D+\frac{h K}{2}\left[x_{0}+2 x_{1}+2 x_{2}+x_{3}\right] .
\end{aligned}
$$

Then the general solution is:

$$
\begin{aligned}
y_{n}=D+\frac{h K}{2}\left[x_{0}+2 x_{1}+2 x_{2}+2 x_{3}+\ldots \ldots .+2 x_{n-1}+x_{n}\right], \\
y_{n}=D+h K\left[\frac{1}{2} x_{0}+\left(x_{0}+h\right)+\left(x_{0}+2 h\right)\right. \\
\left.+\cdots+\left(x_{0}+(n-1) h\right)+\frac{1}{2}\left(x_{0}+n h\right)\right], \\
y_{n}=D+h K\left[\frac{1}{2} x_{0}+\left(x_{0}+h\right)+\left(x_{0}+2 h\right)\right. \\
\left.+\cdots+\left(x_{0}+(n-1) h\right)+\frac{1}{2}\left(x_{0}+n h\right)\right] \\
y_{n}=D+h K \sum_{i=0}^{n-1}\left(x_{0}+i h\right)+\frac{1}{2} n h^{2} K .
\end{aligned}
$$

This can be written in another form:

$$
y_{n}=D+n h K x_{0}+\frac{n^{2} h^{2} K}{2} .
$$

\section{We can prove that:}

1) $\lim _{n \rightarrow \infty} y_{n}=y$

\section{Proof}

Since $\lim _{n \rightarrow \infty} y_{n}=y$ (if and only if) $\lim _{n \rightarrow \infty} E\left|y_{n}-y\right|^{2}=0$

$$
\begin{aligned}
& y_{n}-y=n h K x_{0}+\frac{n^{2}}{2}\left[h^{2} K\right]-\frac{K\left(x^{2}-x_{0}^{2}\right)}{2} \\
& \left|y_{n}-y\right|^{2} \\
& =n^{2} h^{2} K^{2} x_{0}^{2}+2 n h K^{2} x_{0}\left[\frac{n^{2}}{2} h^{2}-\frac{\left(x^{2}-x_{0}^{2}\right)}{2}\right] \\
& +K^{2}\left[\frac{n^{2}}{2} h^{2}-\frac{\left(x^{2}-x_{0}^{2}\right)}{2}\right]^{2}
\end{aligned}
$$

where $h=\frac{x_{n}-x_{0}}{n}$

$$
\begin{aligned}
\left|y_{n}-y\right|^{2}= & K^{2} x_{0}^{2}\left(x_{n}-x_{0}\right)^{2}+K^{2} x_{0}\left(x_{n}-x_{0}\right)\left[\left(x_{n}-x_{0}\right)^{2}-\left(x^{2}-x_{0}^{2}\right)\right] \\
+ & \frac{K^{2}}{4}\left[\left(x_{n}-x_{0}\right)^{2}-\left(x^{2}-x_{0}^{2}\right)\right]^{2} \\
= & K^{2} x_{0}^{2} x_{n}^{2}-2 K^{2} x_{n} x_{0}^{3}+K^{2} x_{0}^{4}+K^{2} x_{n} x_{0}\left(x_{n}-x_{0}\right)^{2}-K^{2} x_{0}^{2}\left(x_{n}-x_{0}\right)^{2} \\
& -K^{2} x_{n} x_{0}\left(x^{2}-x_{0}^{2}\right)+K^{2} x_{0}^{2}\left(x^{2}-x_{0}^{2}\right)+\frac{K^{2}}{4}\left(x_{n}-x_{0}\right)^{4} \\
& -\frac{K^{2}}{2}\left(x_{n}-x_{0}\right)^{2}\left(x^{2}-x_{0}^{2}\right)+\frac{K^{2}}{4}\left(x^{2}-x_{0}^{2}\right)^{2}
\end{aligned}
$$




$$
\begin{aligned}
& \lim _{n \rightarrow \infty} E\left|y_{n}-y\right|^{2} \\
& =18 x_{0}^{2} x^{2}-36 x_{0}^{3} x+18 x_{0}^{4}+18 x x_{0}\left(x-x_{0}\right)^{2}-18 x_{0}^{2}\left(x-x_{0}\right)^{2}-18 x x_{0}\left(x^{2}-x_{0}^{2}\right)+18 x_{0}^{2}\left(x^{2}-x_{0}^{2}\right) \\
& \quad+\frac{18}{4} x^{4}-18 x^{3} x_{0}+27 x^{2} x_{0}^{2}-18 x x_{0}^{3}+\frac{18}{4} x_{0}^{4}-9 x^{4}+18 x^{3} x_{0}-18 x x_{0}^{3}+9 x_{0}^{4}+\frac{18}{4} x^{4}-9 x^{2} x_{0}^{2}+\frac{18}{4} x_{0}^{4} \\
& =18 x_{0}^{2} x^{2}-36 x_{0}^{3} x+18 x_{0}^{4}+72 x x_{0}^{3}-36 x^{2} x_{0}^{2}-36 x_{0}^{4}+\frac{18}{4} x^{4}-18 x^{3} x_{0}+27 x^{2} x_{0}^{2}-18 x x_{0}^{3} \\
& \quad+\frac{18}{4} x_{0}^{4}-9 x^{4}+18 x^{3} x_{0}-18 x x_{0}^{3}+9 x_{0}^{4}+\frac{18}{4} x^{4}-9 x^{2} x_{0}^{2}+\frac{18}{4} x_{0}^{4}
\end{aligned}
$$

i.e.; $\lim _{n \rightarrow \infty} y_{n}=y$.

2) $\lim _{n \rightarrow \infty} E\left\{y_{n}\right\}=E\{y\}$

Verification of Theorem (4.1):

$$
\begin{aligned}
& y_{n}=D+h K \sum_{i=0}^{n-1}\left(x_{0}+i h\right)+\frac{n h^{2} K}{2}=D+h K \sum_{j=1}^{n}\left(x_{0}+(j-1) h\right)+\frac{n h^{2} K}{2} \\
& =D+h K\left[x_{0}+\left(x_{0}+h\right)+\left(x_{0}+2 h\right)+\left(x_{0}+3 h\right)+\cdots\left(x_{0}+(n-1) h\right)\right]+\frac{n h^{2} K}{2} \\
& =D+h K\left[n x_{0}+h[1+2+3+\cdots+(n-1)]\right]+\frac{n h^{2} K}{2} \\
& =D+h K\left[n x_{0}+h\left(\frac{n(n-1)}{2}\right)\right]+\frac{n h^{2} K}{2} \\
& =D+K\left[n h x_{0}+h^{2}\left(\frac{n(n-1)}{2}\right)\right]+\frac{n h^{2} K}{2} \quad \text { where } h=\frac{x_{n}-x_{0}}{n} \\
& =D+K\left[n x_{0} \frac{\left(x_{n}-x_{0}\right)}{n}+\left(\frac{\left(x_{n}-x_{0}\right)}{n}\right)^{2}\left(\frac{n(n-1)}{2}\right)\right]+\frac{\left(x_{n}-x_{0}\right)^{2} K}{2 n} \\
& =D+K\left[x_{0}\left(x_{n}-x_{0}\right)+\frac{(n-1)}{2 n}\left(x_{n}-x_{0}\right)^{2}\right]+\frac{\left(x_{n}-x_{0}\right)^{2} K}{2 n} \\
& E\left(y_{n}\right)=E(D)+E\left\{K\left[x_{0}\left(x_{n}-x_{0}\right)+\frac{(n-1)}{2 n}\left(x_{n}-x_{0}\right)^{2}\right]+\frac{\left(x_{n}-x_{0}\right)^{2} K}{2 n}\right\} \\
& =2+2\left\{E\left(x_{0} x_{n}\right)-E\left(x_{0}^{2}\right)+\frac{(n-1)}{2 n} E\left(x_{n}-x_{0}\right)^{2}\right\}+\frac{2 E\left(x_{n}-x_{0}\right)^{2}}{2 n} \\
& \lim _{n \rightarrow \infty} E\left\{y_{n}\right\}=2+2\left\{E\left(x_{0} x\right)-E\left(x_{0}^{2}\right)+E\left(x-x_{0}\right)^{2} / 2\right\} \\
& =2+2\left\{E\left(x_{0} x\right)-E\left(x_{0}^{2}\right)+\frac{1}{2} E\left(x^{2}\right)-E\left(x_{0} x\right)+\frac{1}{2} E\left(x_{0}^{2}\right)\right\} \\
& =2+E\left(x^{2}\right)-E\left(x_{0}^{2}\right)=E\{y\}
\end{aligned}
$$

i.e.; $\lim _{n \rightarrow \infty} E\left\{y_{n}\right\}=E\{y\}$.

3) $\lim _{n \rightarrow \infty} E\left\{y_{n}^{2}\right\}=E\left\{y^{2}\right\}$
Proof

Since $y_{n}=D+n h K x_{0}+\frac{n^{2} h^{2} K}{2}$ then: 


$$
\begin{aligned}
& E\left\{y_{n}^{2}\right\}=E\left[D+K x_{0}\left(x_{n}-x_{0}\right)+\frac{\left(x_{n}-x_{0}\right)^{2} K}{2}\right]^{2} \\
& =E\left[D+K\left(x_{n}-x_{0}\right) x_{0}\right]^{2}+2 E\left\{\left[D+K\left(x_{n}-x_{0}\right) x_{0}\right]\left[\frac{K\left(x_{n}-x_{0}\right)^{2}}{2}\right]\right\}+E\left[\frac{K\left(x_{n}-x_{0}\right)^{2}}{2}\right]^{2} \\
& =E\left[D^{2}\right]+E\left[K\left(x_{n}-x_{0}\right) x_{0}\right]^{2}+2 E\left[D K\left(x_{n} x_{0}-x_{0}^{2}\right)\right] \\
& +2 E\left\{\left[D+K\left(x_{n}-x_{0}\right) x_{0}\right]\left[\frac{K\left(x_{n}-x_{0}\right)^{2}}{2}\right]\right\}+E\left[\frac{K\left(x_{n}-x_{0}\right)^{2}}{2}\right]^{2} \\
& =E\left[D^{2}\right]+E\left[K^{2}\right] E\left[x_{n} x_{0}\right]^{2}-2 E\left[K^{2}\right] E\left[x_{n} x_{0}^{3}\right]+E\left[K^{2}\right] E\left[x_{0}\right]^{4} \\
& +2 E[D] E[K] E\left[x_{n} x_{0}\right]-2 E[D] E[K] E\left[x_{0}^{2}\right]+2 E[D] E[K] E\left[\left(x_{n}-x_{0}\right)^{2} / 2\right] \\
& +2 E\left[K^{2}\right] E\left[x_{0}\left(x-x_{0}\right)^{3} / 2\right]+E\left[K^{2}\right] E\left[\left(x_{n}-x_{0}\right)^{2} / 2\right]^{2} \\
& =E[D]^{2}+E\left[K^{2}\right] E\left[x_{n} x_{0}\right]^{2}-2 E\left[K^{2}\right] E\left[x_{n} x_{0}^{3}\right]+E\left[K^{2}\right] E\left[x_{0}\right]^{4} \\
& +2 E[D] E[K] E\left[x_{n} x_{0}\right]-2 E[D] E[K] E\left[x_{0}^{2}\right]+E[D] E[K] E\left[\left(x_{n}-x_{0}\right)^{2}\right] \\
& +E\left[K^{2}\right] E\left[x_{0}\left(x_{n}-x_{0}\right)^{3}\right]+\frac{1}{4} E\left[K^{2}\right] E\left[\left(x_{n}-x_{0}\right)^{4}\right] \\
& \lim _{n \rightarrow \infty} E\left\{y_{n}^{2}\right\}=6+6 E\left[x x_{0}\right]^{2}-12 E\left[x x_{0}^{3}\right]+6 E\left[x_{0}\right]^{4}+8 E\left[x x_{0}\right]-8 E\left[x_{0}^{2}\right] \\
& +4 E\left[\left(x-x_{0}\right)^{2}\right]+6 E\left[x_{0}\left(x-x_{0}\right)^{3}\right]+\frac{6}{4} E\left[\left(x-x_{0}\right)^{4}\right] \\
& =6+6 E\left[x x_{0}\right]^{2}-12 E\left[x x_{0}^{3}\right]+6 E\left[x_{0}\right]^{4}+8 E\left[x x_{0}\right]-8 E\left[x_{0}^{2}\right]+4 E\left[x^{2}\right] \\
& -8 E\left[x x_{0}\right]+4 E\left[x_{0}^{2}\right]+6 E\left[x^{3} x_{0}\right]-18 E\left[x^{2} x_{0}^{2}\right]+18 E\left[x x_{0}^{3}\right]-6 E\left[x_{0}^{4}\right] \\
& +\frac{6}{4} E\left[x^{4}\right]-6 E\left[x^{3} x_{0}\right]-6 E\left[x x_{0}^{3}\right]+9 E\left[x^{2} x_{0}^{2}\right]+\frac{6}{4} E\left[x_{0}^{4}\right]=E\left(y^{2}\right)
\end{aligned}
$$

i.e.; $\lim _{n \rightarrow \infty} E\left\{y_{n}^{2}\right\}=E\left\{y^{2}\right\}$.

4) $\lim _{n \rightarrow \infty} \operatorname{Var}\left(y_{n}\right)=\operatorname{Var}(y)$

Proof

$$
\begin{aligned}
\lim _{n \rightarrow \infty} \operatorname{Var}\left\{y_{n}\right\} & =\lim _{n \rightarrow \infty}\left[E\left(y_{n}^{2}\right)-\left[E\left(y_{n}\right)\right]^{2}\right] \\
& =\lim _{n \rightarrow \infty} E\left(y_{n}^{2}\right)-\lim _{n \rightarrow \infty}\left[E\left(y_{n}\right)\right]^{2} \\
& =E\left(y^{2}\right)-[E(y)]^{2}=\operatorname{Var}(y)
\end{aligned}
$$

i.e. $\lim _{n \rightarrow \infty} \operatorname{Var}\left(y_{n}\right)=\operatorname{Var}(y)$

5) $\lim _{n \rightarrow \infty} P D F\left(y_{n}\right)=P D F(y)$

Since $y=D+\frac{K\left(x^{2}-x_{0}^{2}\right)}{2}$

Let us define $Z=D$. Then the inverse transformation is:

$$
D=y-\frac{K\left(x^{2}-x_{0}^{2}\right)}{2} \quad D=z \text { then we have } D=z \text { and }
$$$$
K=\frac{2(y-z)}{x^{2}-x_{0}^{2}}
$$$$
J=\left|\begin{array}{ll}
\frac{\partial K}{\partial y} & \frac{\partial K}{\partial z} \\
\frac{\partial D}{\partial y} & \frac{\partial D}{\partial z}
\end{array}\right|=\left|\begin{array}{cc}
\frac{2}{x^{2}-x_{0}^{2}} & \frac{-2}{x^{2}-x_{0}^{2}} \\
0 & 1
\end{array}\right|=\frac{2}{x^{2}-x_{0}^{2}}
$$

$$
f_{y, z}(y, z)=f_{K, D}\left(\frac{2(y-z)}{x^{2}-x_{0}^{2}}, z\right)|J|=\frac{e^{-4} 2^{Z+\frac{2(Y-Z)}{X^{2}-X_{0}^{2}}}}{z !\left[\frac{2(y-z)}{x^{2}-x_{0}^{2}}\right] !}
$$

Since $D \geq 0$ then $z \geq 0$ this implies 


$$
\begin{gathered}
y \geq z+\frac{K\left(x^{2}-x_{0}^{2}\right)}{2} \\
f_{y}(y)=\sum_{z=0}^{y} \frac{\mathrm{e}^{-4} 2^{z+\frac{2(Y-Z)}{X^{2}-X_{0}^{2}}}}{z !\left[\frac{2(y-z)}{x^{2}-x_{0}^{2}}\right] !}=P D F(y) .
\end{gathered}
$$

Numerically,

since $y_{n}=D+n h K x_{0}+\frac{n^{2} h^{2} K}{2}$

Let $z_{n}=D$ then $K=\frac{\left(y_{n}-z_{n}\right)}{n h x_{0}+\frac{n^{2} h^{2}}{2}}$

$$
\begin{aligned}
J & =\left|\begin{array}{ll}
\frac{\partial k}{\partial y_{n}} & \frac{\partial k}{\partial z_{n}} \\
\frac{\partial D}{\partial y_{n}} & \frac{\partial D}{\partial z_{n}}
\end{array}\right|=\left|\begin{array}{cc}
\frac{1}{n h x_{0}+\frac{n^{2} h^{2}}{2}} & n h x_{0}+\frac{n^{2} h^{2}}{2} \\
0 & 1
\end{array}\right| \\
& =\frac{1}{n h x_{0}+\frac{n^{2} h^{2}}{2}}
\end{aligned}
$$$$
f_{y_{n}, z_{n}}\left(y_{n}, z_{n}\right)=f_{k, D}\left(\frac{\left(y_{n}-z_{n}\right)}{n h x_{0}+\frac{n^{2} h^{2}}{2}}, z_{n}\right)|J|
$$

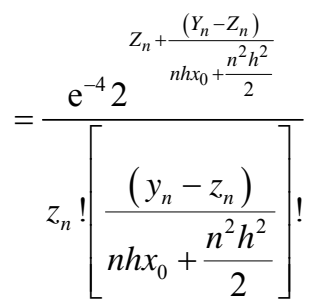

$$
Z_{n}+\frac{\left(Y_{n}-Z_{n}\right)}{n h x_{0}+\frac{n^{2} h^{2}}{2}}
$$

$$
f_{y n}\left(y_{n}\right)=\sum_{z_{n}=0}^{y_{n}} \frac{\mathrm{e}^{-4} 2^{n h x_{0}+\frac{n^{2} h^{2}}{2}}}{z_{n} !\left[\frac{\left(y_{n}-z_{n}\right)}{n h x_{0}+\frac{n^{2} h^{2}}{2}}\right] !} \text { where } h=\frac{x_{n}-x_{0}}{n}
$$

$$
\begin{aligned}
f_{y n}\left(y_{n}\right) & =\sum_{z_{n}}^{y_{n}} \frac{\mathrm{e}^{-4} 2^{Z_{n}+\frac{\left(y_{n}-Z_{n}\right)}{\left(x_{n}-x_{0}\right) x_{0}+\left(x_{n}-x_{0}\right)^{2} / 2}}}{z_{n} !\left[\frac{\left(y_{n}-z_{n}\right)}{\left(x_{n}-x_{0}\right) x_{0}+\left(x_{n}-x_{0}\right)^{2} / 2}\right] !} \\
& =P D F\left(y_{n}\right)
\end{aligned}
$$

$$
\lim _{n \rightarrow \infty} f_{y_{n}}\left(y_{n}\right)=\sum_{z=0}^{y} \frac{\mathrm{e}^{-4} 2^{Z+\frac{2(Y-Z)}{X^{2}-X_{0}^{2}}}}{z !\left[\frac{2(y-z)}{x^{2}-x_{0}^{2}}\right] !}=f_{y}(y)
$$

i.e.; $\lim _{n \rightarrow \infty} \operatorname{PDF}\left(y_{n}\right)=\operatorname{PDF}(y)$.

\section{Example (5.2)}

Solve the problem

$$
\frac{\mathrm{d} y}{\mathrm{~d} t}=y^{2}-K y, y(0)=K, t \in\left[0, t_{n}\right], K \sim \exp (1)
$$

The exact solution

$$
y=K
$$

The numerical solution by the Euler method:

$$
y_{n}=y_{n-1}+h f\left(y_{n-1}, t_{n-1}\right), y(0)=K .
$$

$\operatorname{AT} n=1$

$$
y_{1}=y_{0}+h f\left(y_{0}, t_{0}\right)=K+h\left(y_{0}^{2}-K y_{0}\right)=K .
$$

At $n=2$

$$
y_{2}=y_{1}+h f\left(y_{1}, t_{1}\right)=K+h\left(y_{1}^{2}-k y_{1}\right)=K .
$$

At $n=3$

$$
y_{3}=y_{2}+h f\left(y_{2}, t_{2}\right)=K+h\left(y_{2}^{2}-k y_{2}\right)=K .
$$

And so on....

Then the general numerical solution: $y_{n}=K$. It is clear that:

1) $\lim _{n \rightarrow \infty} y_{n}=y$

Since $\lim _{n \rightarrow \infty} E\left|y_{n}-y\right|^{2}=\lim _{n \rightarrow \infty} E|K-K|^{2}=0$

Verification of Theorem (4.1)

It is clear that:

2) $\lim _{n \rightarrow \infty} E\left\{y_{n}\right\}=E\{y\}$

$$
E\left(y_{n}\right)=E(K)=K=E(y)
$$

3) $\lim _{n \rightarrow \infty} E\left\{y_{n}^{2}\right\}=E\left\{y^{2}\right\}$

Since $y_{n}=K \Rightarrow E\left\{y_{n}^{2}\right\}=E[K]^{2}=K^{2}=E\left\{y^{2}\right\}$

4) $\lim _{n \rightarrow \infty} \operatorname{Var}\left(y_{n}\right)=\operatorname{Var}(y)$

$$
\begin{aligned}
\lim _{n \rightarrow \infty} \operatorname{Var}\left\{y_{n}\right\} & =\lim _{n \rightarrow \infty}\left[E\left(y_{n}^{2}\right)-\left[E\left(y_{n}\right)\right]^{2}\right] \\
& =\lim _{n \rightarrow \infty} E\left(y_{n}^{2}\right)-\lim _{n \rightarrow \infty}\left[E\left(y_{n}\right)\right]^{2} \\
& =E\left(y^{2}\right)-[E(y)]^{2}=\operatorname{Var}(y)
\end{aligned}
$$

5) $\lim _{n \rightarrow \infty} \operatorname{PDF}\left(y_{n}\right)=\operatorname{PDF}(y)$

$y=K$ Then $|J|=1$ which implies

$$
\operatorname{PDF}(y)=|J| \operatorname{PDF}(k)=\mathrm{e}^{-y}
$$

$y_{n}=K$ Then $|J|=1 \quad$ which implies

$$
\operatorname{PDF}\left(y_{n}\right)=|J| P D F(K)=\mathrm{e}^{-y_{n} k}
$$


Then: $\lim _{n \rightarrow \infty} \operatorname{PDF}\left(y_{n}\right)=\operatorname{PDF}(y)$

\section{Conclusions}

The initially valued first order random differential equations can be solved numerically using the random Euler and random Runge-Kutta methods in mean square sense. The existence and uniqueness of the solution have been proved. The convergence of the presented numerical techniques has been proven in mean square sense. The results of the paper have been illustrated through some examples.

\section{References}

[1] K. Burrage, and P.M. Burrage, "High Strong Order Explicit Runge-Kutta Methods for Stochastic Ordinary Differential Equations," Applied Numerical Mathematics, Vol. 22, No. 1-3, 1996, pp. 81-101. doi:10.1016/S0168-9274(96)00027-X

[2] K. Burrage, and P. M. Burrage, "General Order Conditions for Stochastic Runge-Kutta Methods for Both Commuting and Non-Commuting Stochastic Ordinary Equations," Applied Numerical Mathematics, Vol. 28, No. 2-4, 1998, pp. 161-177. doi:10.1016/S0168-9274(98)00042-7

[3] J. C. Cortes, L. Jodar and L. Villafuerte, "Numerical Soluion of Random Differential Equations, a Mean Square Approach," Mathematical and Computer Modelling, Vol. 45, No.7, 2007, pp. 757-765. doi:10.1016/j.mcm.2006.07.017

[4] J. C. Cortes, L. Jodar, and L.Villafuerte, "A Random
Euler Method for Solving Differential Equations with Uncertainties," Progress in Industrial Mathematics at ECMI, Madrid, 2006.

[5] H. Lamba, J. C. Mattingly and A. Stuart, "An adaptive Euler-Maruyama Scheme for SDEs, Convergence and Stability," IMA Journal of Numerical Analysis, Vol. 27, No. 3, 2007, pp. 479-506. doi:10.1093/imanum/dr1032

[6] E. Platen, "An Introduction to Numerical Methods for Stochastic Differential Equations," Acta Numerica, Vol. 8, 1999, pp. 197-246. doi:10.1017/S0962492900002920

[7] D. J. Higham, "An Algorithmic Introduction to Numerical Simulation of SDE," SIAM Review, Vol. 43, No. 3, 2001, pp. 525-546. doi:10.1137/S0036144500378302

[8] D. Talay, and L. Tubaro, "Expansion of The Global Error for Numerical Schemes Solving Stochastic Differential Equation," Stochastic Analysis and Applications, Vol. 38, No. 4, 1990, pp. 483-509. doi:10.1080/07362999008809220

[9] P. M. Burrage, "Numerical Methods for SDE," Ph.D. Thesis, The University of Queensland, 1999.

[10] P. E. Kloeden, E. Platen and H. Schurz, "Numerical Solution of SDE Through Computer Experiments," Second Edition, Springer, 1997.

[11] M. A. El-Tawil, "The Approximate Solutions of Some Stochastic Differential Equations Using Transformation," Journal of Applied Mathematics and Computing, Vol. 164, No. 1, 2005, pp. 167-178. doi:10.1016/j.amc.2004.04.062

[12] P. E. Kloeden, and E. Platen, "Numeical Solution of Stochastic Differential Equations," Springer, Berlin, 1999.

[13] T. T. Soong, "Random Differential Equations in Science and Engineering," Academic Press, New York, 1973. 